\title{
SYNTHESIS AND CHARACTERIZATION OF MIXED LIGAND COM PLEXES OF ZIRCONIUM(IV) WITH SULPHUR, NITROGEN AND OXYGEN DONOR LIGANDS
}

\author{
Abhishek Srivastava ${ }^{1,}{ }^{*}$, Neetu Srivastava ${ }^{2}$, Umesh $N$ athTripathi ${ }^{2}$, Afshan Siddiqui ${ }^{2}$
}

https://doi.org/10.23939/chcht13.01.023

\begin{abstract}
A new route to synthesize new nanosized mixed ligand complexes of $\mathrm{Zr}(\mathrm{IV})$ have been investigated by the reaction of zirconium(IV) chloride with 3(2'-hydroxyphenyl)-5-(4-substituted phenyl)pyrazolines and ammonium salts of dithiophosphate, which after being treated with $\mathrm{H}_{2} \mathrm{~S}$ gas acts as a precursor for $\mathrm{ZrS}_{2}$. These complexes have been examined for crystalline/amorphous nature through XRD. The morphology of the complexes was studied by employing XRD which shows that all the complexes are amorphous solid. Molecular weight measurements, elemental analysis in conjugation with spectroscopic (IR, ${ }^{1} \mathrm{H}$ NMR, ${ }^{31} \mathrm{P}$ NMR) studies reveal dimeric nature of the complexes, in which dithiophosphate and pyrazoline are bidentate. Scanning electron microscopic images indicate that the particles are in a nano range. Putting all the facts together, coordination number six is proposed for zirconium with octahedral geometry.
\end{abstract}

Keywords: zirconium(IV), dithiophosphate, pyrazoline, nanoparticle, sol-gel.

\section{Introduction}

Metal organic frameworks are made by linking metal and organic units by strong bonds [1-3]. Since most of the zirconium complexes are sensitive to hydrolysis, the metal organic frameworks of $\mathrm{Zr}(\mathrm{IV})$ complexes have been developed in anhydrous medium by the interaction of zirconium tetrachloride and various mixed ligands in different stoichiometric ratio [4-6]. The $\mathrm{Zr}(\mathrm{IV})$ complexes with nitrogen and sulphur donor ligands have received considerable attention due to their wide spread utilization as an effective precursor for making $\mathrm{ZrO}_{2}$ and $\mathrm{ZrS}_{2}$ by MOCVD technique [6-10].

\footnotetext{
${ }^{1}$ G.L.A. University, Chaumuhan, Mathura 281406, U.P., India

${ }^{2}$ Deen Dayal Upadhyaya Gorakhpur University, Gorakhpur 273001, U.P., India

*aabhichem@gla.ac.in

(c) Srivastava A., Srivastava N., Tripathi U., Siddiqui A., 2019
}

It is well known that mixed ligand complexes can exhibit superior properties as compared to their pure counterpart. Biological activity of zirconium mixed ligand complexes were tested against various pathogenic microorganisms [11-14]. Mixed ligand complexes of $\mathrm{Zr}(\mathrm{IV})$ have been extensively used as a catalyst in various polymerization reactions [15-19]. The metal organic framework of $\mathrm{Zr}(\mathrm{IV})$ complexes acts as a precursor for the development of $\mathrm{ZrO}_{2}$ and $\mathrm{ZrS}_{2}$. $\mathrm{ZrO}_{2}$ exhibits wide range of applications in ceramics [20-21], chromatography [22-23] and pharmaceuticals [24-25]. By mixing $\mathrm{ZrO}_{2}$ with other metallic oxides such as $\mathrm{MgO}, \mathrm{CaO}$ and $\mathrm{Y}_{2} \mathrm{O}_{3}$, great molecular stability can be obtained [26]. Yttrium- stabilized zirconia also known as tetragonal zirconia polycrystals (TPZ) is presently the most studied combination for biomedical ceramics that can be used in manufacturing of ball heads for total hip replacements (THR) [27-28]. $\mathrm{ZrS}_{2}$ nanoparticles have found application in the manufacturing of high-performance Schottky solar cells and nano discs [29-30].

A few complexes of $\mathrm{Zr}(\mathrm{IV})$ with pyrazolines ligands have been reported till now [31-32]. The wide range application of nanosized $\mathrm{ZrO}_{2}$ and $\mathrm{ZrS}_{2}$ has drawn our attention in developing a MOFs of $\mathrm{Zr}(\mathrm{IV})$ complexes with dithiophosphate and pyrazolines ligands, which can act as a precursor for the synthesis of nano range $\mathrm{ZrS}_{2}$.

The objective of the work is to synthesize and characterize some new mixed ligand complexes of $\mathrm{Zr}(\mathrm{IV})$.

\section{Experimental}

\subsection{Material and Methods}

All reactions were carried out under absolutely dry conditions. Solvents were distilled, dried and purified by standard techniques [33]. Pyrazolines and ammonium salt of dialkyl/alkylene dithiophosphates 
were prepared by method described in [34, 35]. Chlorine was estimated by Volhard's method [34] and zirconium was estimated as zirconium phosphate [33]. The elemental analysis $(\mathrm{C}, \mathrm{H}, \mathrm{N})$ were obtained by using a Coleman CHN analyzer (Table 1). IR spectra were recorded on Varian 3100 FT-IR spectrophotometer in the range of $4000-200 \mathrm{~cm}^{-1}$. ${ }^{1} \mathrm{H}$ NMR spectra and proton decoupled ${ }^{13} \mathrm{C}$ NMR spectra were recorded at room temperature on JOEL AL 300 FT NMR spectrophotometer operated at $300.40 \mathrm{MHz}$. The $\mathrm{X}$-ray diffraction studies were carried out on Bruker Nonious Kappa CCD diffractometer at room temperature. SEM studies have been carried out on JEOL 2010 high-resolution scanning electron microscope, operated at $200 \mathrm{keV}$. The FAB mass spectra were recorded on JOEL SX102 mass spectrometer using Argon or Xenon $(6 \mathrm{kV}, 10 \mathrm{~mA})$ as the FAB gas.

\subsection{Synthesis of $\mathrm{ZrCl}_{2}\left(\mathrm{C}_{15} \mathrm{H}_{12} \mathrm{~N}_{2} \mathrm{OH}\right)\left(\mathrm{OC}_{3} \mathrm{H}_{7}\right)_{2} \mathrm{PS}_{2}$}

A benzene solution of pyrazoline $(1.09 \mathrm{~g}$, $4.60 \mathrm{mmol}$ ) was added drop-wise to the suspension of zirconium tetrachloride $(1.07 \mathrm{~g}, 4.60 \mathrm{mmol})$ under constant stirring at room temperature. The reaction mixture was stirred for $2-3 \mathrm{~h}$ to ensure the completion of reaction. The solution of dithiophosphate in methanol $(0.97 \mathrm{~g}, 4.60 \mathrm{mmol})$ was added drop-wise under constant stirring to the same reaction mixture and again stirred for
3-4 h. The by-product $\left(\mathrm{NH}_{4} \mathrm{Cl}\right)$ was filtered off through alkoxy funnel and the volatiles were removed from the filtrate under reduced pressure. A reddish brown colored solid was thus obtained. The remaining compounds were prepared by the same procedure.

\subsection{Synthesis of $\mathrm{Zr}_{2}\left(\mathrm{C}_{15} \mathrm{H}_{12} \mathrm{~N}_{2} \mathrm{OH}\right)_{2}\left(\mathrm{OC}_{3} \mathrm{H}_{7}\right)_{4} \mathrm{P}_{2} \mathrm{~S}_{6}$}

Dry $\mathrm{H}_{2} \mathrm{~S}$ gas was bubbled in the methanolic solution of zirconium complex. As the bubbling started, the formation of white colored precipitate was observed. The bubbling of the gas was continued for $2 \mathrm{~h}$ to ensure complete precipitation. This reaction mixture was filtered through alkoxy funnel and the precipitate was analyzed as the final product. The remaining compounds (1-24) were prepared by the same procedure.

\section{Results and Discussion}

All the compounds are white colored solids, hygroscopic and stable at room temperature. They are soluble in common organic solvents (methanol, benzene and chloroform) and coordinating solvents (THF, DMSO and DMF). The elemental analysis $(\mathrm{C}, \mathrm{H}$, $\mathrm{S}, \mathrm{Cl}, \mathrm{Zr}$ and $\mathrm{N}$ ) data (Table 1) is in accordance with the stoichiometry proposed for respective compounds.

Table 1

Analytical data for $\mathrm{Zr}_{2}\left(\mathrm{C}_{15} \mathrm{H}_{12} \mathrm{~N}_{2} \mathrm{OX}\right)_{2}\left[\mathrm{~S}_{6} \mathrm{P}_{2}(\mathrm{OR})_{4}\right]$

\begin{tabular}{|c|c|c|c|c|c|c|c|}
\hline \multirow{2}{*}{ No. } & \multirow{2}{*}{ Compound } & \multirow{2}{*}{$\begin{array}{c}\text { Molecular } \\
\text { weight } \\
\text { Found } \\
\text { (Calcd) }\end{array}$} & \multicolumn{5}{|c|}{$\begin{array}{c}\text { Analysis } \\
\text { Found (Calcd.) }\end{array}$} \\
\hline & & & $\mathrm{C}$ & $\mathrm{H}$ & $\mathrm{N}$ & $S$ & Metal \\
\hline 1 & 2 & 3 & 4 & 5 & 6 & 7 & 8 \\
\hline 1 & $\mathrm{Zr}_{2}\left(\mathrm{C}_{15} \mathrm{H}_{12} \mathrm{~N}_{2} \mathrm{OH}\right)_{2}\left[\mathrm{~S}_{6} \mathrm{P}_{2}\left(\mathrm{OCH}_{2} \mathrm{CH}_{2} \mathrm{CH}_{3}\right)_{4}\right]$ & $\begin{array}{c}1140 \\
(1146.4)\end{array}$ & $\begin{array}{c}43.92 \\
(43.96)\end{array}$ & $\begin{array}{c}3.80 \\
(3.83)\end{array}$ & $\begin{array}{c}2.45 \\
(2.44)\end{array}$ & $\begin{array}{c}16.70 \\
(16.75)\end{array}$ & $\begin{array}{c}15.87 \\
(15.91)\end{array}$ \\
\hline 2 & $\mathrm{Zr}_{2}\left(\mathrm{C}_{15} \mathrm{H}_{12} \mathrm{~N}_{2} \mathrm{OH}\right)_{2}\left[\mathrm{~S}_{6} \mathrm{P}_{2}\left(\mathrm{OC}_{6} \mathrm{H}_{5}\right)_{4}\right]$ & $\begin{array}{c}1280 \\
(1282.4)\end{array}$ & $\begin{array}{c}41.10 \\
(41.17)\end{array}$ & $\begin{array}{c}3.18 \\
(3.21) \\
\end{array}$ & $\begin{array}{c}2.16 \\
(2.18)\end{array}$ & $\begin{array}{l}14.95 \\
(14.97)\end{array}$ & $\begin{array}{r}14.20 \\
(14.22) \\
\end{array}$ \\
\hline 3 & $\mathrm{Zr}_{2}\left(\mathrm{C}_{15} \mathrm{H}_{12} \mathrm{~N}_{2} \mathrm{OH}\right)_{2}\left[\mathrm{~S}_{6} \mathrm{P}_{2}\left\{\mathrm{OC}\left(\mathrm{CH}_{3}\right)_{2} \mathrm{CH}_{2} \mathrm{CH}\left(\mathrm{CH}_{3}\right) \mathrm{O}\right\}_{2}\right]$ & $\begin{array}{c}1150 \\
(1142.4) \\
\end{array}$ & $\begin{array}{c}44.05 \\
(44.12)\end{array}$ & $\begin{array}{c}3.85 \\
(3.86) \\
\end{array}$ & $\begin{array}{c}2.43 \\
(2.45) \\
\end{array}$ & $\begin{array}{c}16.74 \\
(16.81)\end{array}$ & $\begin{array}{r}15.92 \\
(15.97) \\
\end{array}$ \\
\hline 4 & $\mathrm{Zr}_{2}\left(\mathrm{C}_{15} \mathrm{H}_{12} \mathrm{~N}_{2} \mathrm{OH}\right)_{2}\left[\mathrm{~S}_{6} \mathrm{P}_{2}\left\{\mathrm{OCH}_{2} \mathrm{C}\left(\mathrm{CH}_{3}\right)_{2} \mathrm{CH}_{2} \mathrm{O}\right\}_{2}\right]$ & $\begin{array}{c}1108 \\
(1114.4) \\
\end{array}$ & $\begin{array}{c}42.96 \\
(43.07) \\
\end{array}$ & $\begin{array}{c}3.85 \\
(3.87) \\
\end{array}$ & $\begin{array}{c}2.50 \\
(2.51) \\
\end{array}$ & $\begin{array}{c}17.20 \\
(17.23)\end{array}$ & $\begin{array}{r}16.35 \\
(16.37) \\
\end{array}$ \\
\hline 5 & $\left.\mathrm{Zr}_{2}\left(\mathrm{C}_{15} \mathrm{H}_{12} \mathrm{~N}_{2} \mathrm{OH}\right)_{2}\left[\mathrm{~S}_{6} \mathrm{P}_{21} \mathrm{OC}\left(\mathrm{CH}_{3}\right)_{2} \mathrm{C}\left(\mathrm{CH}_{3}\right)_{2} \mathrm{O}\right\}_{2}\right]$ & $\begin{array}{c}1140 \\
(1142.4) \\
\end{array}$ & $\begin{array}{c}44.06 \\
(44.12) \\
\end{array}$ & $\begin{array}{c}3.84 \\
(3.86) \\
\end{array}$ & $\begin{array}{c}2.45 \\
(2.45) \\
\end{array}$ & $\begin{array}{c}16.77 \\
(16.81)\end{array}$ & $\begin{array}{r}16.00 \\
(15.97) \\
\end{array}$ \\
\hline 6 & $\mathrm{Zr}_{2}\left(\mathrm{C}_{15} \mathrm{H}_{12} \mathrm{~N}_{2} \mathrm{OH}\right)_{2}\left[\mathrm{~S}_{6} \mathrm{P}_{2}\left\{\mathrm{OCH} 2 \mathrm{CH} 2 \mathrm{CH}\left(\mathrm{CH}_{3}\right) \mathrm{O}\right\}_{2}\right]$ & $\begin{array}{c}1085 \\
(1083.4) \\
\end{array}$ & $\begin{array}{c}4.00 \\
(42.09) \\
\end{array}$ & $\begin{array}{c}3.85 \\
(3.88) \\
\end{array}$ & $\begin{array}{c}2.56 \\
(2.58) \\
\end{array}$ & $\begin{array}{l}17.70 \\
(17.72)\end{array}$ & $\begin{array}{r}16.80 \\
(16.84) \\
\end{array}$ \\
\hline 7 & $\mathrm{Zr}_{2}\left(\mathrm{C}_{15} \mathrm{H}_{12} \mathrm{~N}_{2} \mathrm{OCH}_{3}\right)_{2}\left[\mathrm{~S}_{6} \mathrm{P}_{2}\left(\mathrm{OCH}_{2} \mathrm{CH}_{2} \mathrm{CH}_{3}\right)_{4}\right]$ & $\begin{array}{c}1170 \\
(1174.4)\end{array}$ & $\begin{array}{c}44.85 \\
(44.96) \\
\end{array}$ & $\begin{array}{c}3.80 \\
(3.83) \\
\end{array}$ & $\begin{array}{c}2.37 \\
(2.38) \\
\end{array}$ & $\begin{array}{l}16.30 \\
(16.35) \\
\end{array}$ & $\begin{array}{r}15.50 \\
(15.53) \\
\end{array}$ \\
\hline 8 & $\mathrm{Zr}_{2}\left(\mathrm{C}_{15} \mathrm{H}_{12} \mathrm{~N}_{2} \mathrm{OCH}_{3}\right)_{2}\left[\mathrm{~S}_{6} \mathrm{P}_{2}\left(\mathrm{OC}_{6} \mathrm{H}_{5}\right)_{4}\right]$ & $\begin{array}{c}1310 \\
(1310.4)\end{array}$ & $\begin{array}{c}42.04 \\
(42.12)\end{array}$ & $\begin{array}{c}3.15 \\
(3.21)\end{array}$ & $\begin{array}{c}2.12 \\
(2.14)\end{array}$ & $\begin{array}{c}14.61 \\
(14.65)\end{array}$ & $\begin{array}{r}13.93 \\
(13.92)\end{array}$ \\
\hline 9 & $\mathrm{Zr}_{2}\left(\mathrm{C}_{15} \mathrm{H}_{12} \mathrm{~N}_{2} \mathrm{OCH}_{3}\right)_{2}\left[\mathrm{~S}_{6} \mathrm{P}_{2}\left\{\mathrm{OC}\left(\mathrm{CH}_{3}\right)_{2} \mathrm{CH}_{2} \mathrm{CH}\left(\mathrm{CH}_{3}\right) \mathrm{O}\right\}_{2}\right]$ & $\begin{array}{c}1175 \\
(1170.4)\end{array}$ & $\begin{array}{c}45.05 \\
(45.11)\end{array}$ & $\begin{array}{c}3.80 \\
(3.85)\end{array}$ & $\begin{array}{c}2.36 \\
(2.39)\end{array}$ & $\begin{array}{c}16.36 \\
(16.40)\end{array}$ & $\begin{array}{c}15.55 \\
(15.58)\end{array}$ \\
\hline
\end{tabular}


Synthesis and Characterization of M ixed Ligand Complexes of Zirconium(IV) with Sulphur...

Table 1 (continued)

\begin{tabular}{|c|c|c|c|c|c|c|c|}
\hline 1 & 2 & 3 & 4 & 5 & 6 & 7 & 8 \\
\hline 10 & $\mathrm{Zr}_{2}\left(\mathrm{C}_{15} \mathrm{H}_{12} \mathrm{~N}_{2} \mathrm{OCH}_{3}\right)_{2}\left[\mathrm{~S}_{6} \mathrm{P}_{2}\left\{\mathrm{OCH}_{2} \mathrm{C}\left(\mathrm{CH}_{3}\right)_{2} \mathrm{CH}_{2} \mathrm{O}\right\}_{2}\right]$ & 1140 (1142.4) & $\begin{array}{c}44.05 \\
(44.12) \\
\end{array}$ & $\begin{array}{c}3.85 \\
(3.86) \\
\end{array}$ & $\begin{array}{c}2.43 \\
(2.45) \\
\end{array}$ & $\begin{array}{c}16.75 \\
(16.81) \\
\end{array}$ & $\begin{array}{c}15.94 \\
(15.97) \\
\end{array}$ \\
\hline 11 & $\mathrm{Zr}_{2}\left(\mathrm{C}_{15} \mathrm{H}_{12} \mathrm{~N}_{2} \mathrm{OCH}_{3}\right)_{2}\left[\mathrm{~S}_{6} \mathrm{P}_{2}\left\{\mathrm{OC}\left(\mathrm{CH}_{3}\right)_{2} \mathrm{C}\left(\mathrm{CH}_{3}\right)_{2} \mathrm{O}\right\}_{2}\right]$ & $1175(1170.4)$ & $\begin{array}{c}45.06 \\
(45.11)\end{array}$ & $\begin{array}{c}3.83 \\
(3.85) \\
\end{array}$ & $\begin{array}{c}2.37 \\
(2.39) \\
\end{array}$ & $\begin{array}{c}16.35 \\
(16.40)\end{array}$ & $\begin{array}{c}15.55 \\
(15.58) \\
\end{array}$ \\
\hline 12 & $\mathrm{Zr}_{2}\left(\mathrm{C}_{15} \mathrm{H}_{12} \mathrm{~N}_{2} \mathrm{OCH}_{3}\right)_{2}\left[\mathrm{~S}_{6} \mathrm{P}_{2}\left\{\mathrm{OCH}_{2} \mathrm{CH}_{2} \mathrm{CH}\left(\mathrm{CH}_{3}\right) \mathrm{O}\right\}_{2}\right]$ & $1110(1111.4)$ & $\begin{array}{c}43.10 \\
(43.19) \\
\end{array}$ & $\begin{array}{c}3.87 \\
(3.89) \\
\end{array}$ & $\begin{array}{c}2.51 \\
(2.52) \\
\end{array}$ & $\begin{array}{c}17.22 \\
(17.28) \\
\end{array}$ & $\begin{array}{c}16.37 \\
(16.41) \\
\end{array}$ \\
\hline 13 & $\mathrm{Zr}_{2}\left(\mathrm{C}_{15} \mathrm{H}_{12} \mathrm{~N}_{2} \mathrm{O}_{2} \mathrm{CH}_{3}\right)_{2}\left[\mathrm{~S}_{6} \mathrm{P}_{2}\left(\mathrm{OCH}_{2} \mathrm{CH}_{2} \mathrm{CH}_{3}\right)_{4}\right]$ & 1208 (1206.4) & $\begin{array}{c}43.68 \\
(43.77) \\
\end{array}$ & $\begin{array}{c}3.60 \\
(3.63) \\
\end{array}$ & $\begin{array}{c}2.30 \\
(2.32) \\
\end{array}$ & $\begin{array}{c}15.90 \\
(15.92)\end{array}$ & $\begin{array}{c}15.09 \\
(15.12) \\
\end{array}$ \\
\hline 14 & $\mathrm{Zr}_{2}\left(\mathrm{C}_{15} \mathrm{H}_{12} \mathrm{~N}_{2} \mathrm{O}_{2} \mathrm{CH}_{3}\right)_{2}\left[\mathrm{~S}_{6} \mathrm{P}_{2}\left(\mathrm{OC}_{6} \mathrm{H}_{5}\right)_{4}\right]$ & 1345 (1342.4) & $\begin{array}{c}50.00 \\
(50.06)\end{array}$ & $\begin{array}{c}3.71 \\
(3.73)\end{array}$ & $\begin{array}{l}2.07 \\
(2.09)\end{array}$ & $\begin{array}{c}14.28 \\
(14.30)\end{array}$ & $\begin{array}{c}13.55 \\
(13.59)\end{array}$ \\
\hline 15 & $\mathrm{Zr}_{2}\left(\mathrm{C}_{15} \mathrm{H}_{12} \mathrm{~N}_{2} \mathrm{O}_{2} \mathrm{CH}_{3}\right)_{2}\left[\mathrm{~S}_{6} \mathrm{P}_{2}\left\{\mathrm{OC}\left(\mathrm{CH}_{3}\right)_{2} \mathrm{CH}_{2} \mathrm{CH}\left(\mathrm{CH}_{3}\right) \mathrm{O}\right\}_{2}\right]$ & 1255 (1248.4) & $\begin{array}{c}42.25 \\
(42.29) \\
\end{array}$ & $\begin{array}{c}3.36 \\
(3.39) \\
\end{array}$ & $\begin{array}{c}2.23 \\
(2.24) \\
\end{array}$ & $\begin{array}{c}15.36 \\
(15.38) \\
\end{array}$ & $\begin{array}{c}14.59 \\
(14.61) \\
\end{array}$ \\
\hline 16 & $\mathrm{Zr}_{2}\left(\mathrm{C}_{15} \mathrm{H}_{12} \mathrm{~N}_{2} \mathrm{O}_{2} \mathrm{CH}_{3}\right)_{2}\left[\mathrm{~S}_{6} \mathrm{P}_{2}\left\{\mathrm{OCH}_{2} \mathrm{C}\left(\mathrm{CH}_{3}\right)_{2} \mathrm{CH}_{2} \mathrm{O}\right\}_{2}\right]$ & $1120(1129.4)$ & $\begin{array}{c}44.56 \\
(44.63)\end{array}$ & $\begin{array}{c}3.92 \\
(3.95)\end{array}$ & $\begin{array}{l}2.47 \\
(2.48)\end{array}$ & $\begin{array}{c}16.95 \\
(17.00)\end{array}$ & $\begin{array}{c}16.10 \\
(16.15)\end{array}$ \\
\hline 17 & $\mathrm{Zr}_{2}\left(\mathrm{C}_{15} \mathrm{H}_{12} \mathrm{~N}_{2} \mathrm{O}_{2} \mathrm{CH}_{3}\right)_{2}\left[\mathrm{~S}_{6} \mathrm{P}_{2}\left\{\mathrm{OC}\left(\mathrm{CH}_{3}\right)_{2} \mathrm{C}\left(\mathrm{CH}_{3}\right)_{2} \mathrm{O}\right\}_{2}\right]$ & 1245 (1248.4) & $\begin{array}{c}42.19 \\
(42.29) \\
\end{array}$ & $\begin{array}{c}3.37 \\
(3.39) \\
\end{array}$ & $\begin{array}{c}2.20 \\
(2.24) \\
\end{array}$ & $\begin{array}{c}15.35 \\
(15.38) \\
\end{array}$ & $\begin{array}{r}14.56 \\
(14.61) \\
\end{array}$ \\
\hline 18 & 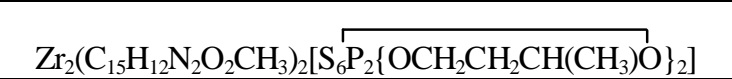 & 1095 (1098.4) & $\begin{array}{c}43.62 \\
(43.70)\end{array}$ & $\begin{array}{c}3.95 \\
(3.98)\end{array}$ & $\begin{array}{l}2.53 \\
(2.55)\end{array}$ & $\begin{array}{c}17.46 \\
(17.48)\end{array}$ & $\begin{array}{c}16.58 \\
(16.61)\end{array}$ \\
\hline 19 & $\mathrm{Zr}_{2}\left(\mathrm{C}_{15} \mathrm{H}_{12} \mathrm{~N}_{2} \mathrm{OCl}\right)_{2}\left[\mathrm{~S}_{6} \mathrm{P}_{2}\left(\mathrm{OCH}_{2} \mathrm{CH}_{2} \mathrm{CH}_{3}\right)_{4}\right]$ & 1215 (1215.4) & $\begin{array}{c}26.63 \\
(26.66) \\
\end{array}$ & $\begin{array}{c}2.18 \\
(2.19) \\
\end{array}$ & $\begin{array}{c}2.29 \\
(2.30) \\
\end{array}$ & $\begin{array}{c}15.77 \\
(15.80) \\
\end{array}$ & $\begin{array}{c}14.96 \\
(15.01) \\
\end{array}$ \\
\hline 20 & $\mathrm{Zr}_{2}\left(\mathrm{C}_{15} \mathrm{H}_{12} \mathrm{~N}_{2} \mathrm{OCl}\right)_{2}\left[\mathrm{~S}_{6} \mathrm{P}_{2}\left(\mathrm{OC}_{6} \mathrm{H}_{5}\right)_{4}\right]$ & $1352(1351.4)$ & $\begin{array}{c}34.60 \\
(34.63)\end{array}$ & $\begin{array}{c}2.55 \\
(2.56) \\
\end{array}$ & $\begin{array}{c}2.05 \\
(2.07)\end{array}$ & $\begin{array}{c}14.17 \\
(14.21)\end{array}$ & $\begin{array}{c}13.45 \\
(13.50) \\
\end{array}$ \\
\hline 21 & $\mathrm{Zr}_{2}\left(\mathrm{C}_{15} \mathrm{H}_{12} \mathrm{~N}_{2} \mathrm{OCl}\right)_{2}\left[\mathrm{~S}_{6} \mathrm{P}_{2}\left\{\mathrm{OC}\left(\mathrm{CH}_{3}\right)_{2} \mathrm{CH}_{2} \mathrm{CH}\left(\mathrm{CH}_{3}\right) \mathrm{O}\right\}_{2}\right]$ & $1220(1211.4)$ & $\begin{array}{c}26.72 \\
(26.75) \\
\end{array}$ & $\begin{array}{c}2.20 \\
(2.21) \\
\end{array}$ & $\begin{array}{c}2.28 \\
(2.31) \\
\end{array}$ & $\begin{array}{c}15.80 \\
(15.85) \\
\end{array}$ & $\begin{array}{c}15.03 \\
(15.06) \\
\end{array}$ \\
\hline 22 & $\mathrm{Zr}_{2}\left(\mathrm{C}_{15} \mathrm{H}_{12} \mathrm{~N}_{2} \mathrm{OCl}\right)_{2}\left[\mathrm{~S}_{6} \mathrm{P}_{2}\left\{\mathrm{OCH}_{2} \mathrm{C}\left(\mathrm{CH}_{3}\right)_{2} \mathrm{CH}_{2} \mathrm{O}\right\}_{2}\right]$ & $1185(1183.4)$ & $\begin{array}{c}25.30 \\
(25.35)\end{array}$ & $\begin{array}{c}2.12 \\
(2.14)\end{array}$ & $\begin{array}{l}2.35 \\
(2.37)\end{array}$ & $\begin{array}{c}16.18 \\
(16.22)\end{array}$ & $\begin{array}{c}15.37 \\
(15.41) \\
\end{array}$ \\
\hline 23 & $\mathrm{Zr}_{2}\left(\mathrm{C}_{15} \mathrm{H}_{12} \mathrm{~N}_{2} \mathrm{OCl}\right)_{2}\left[\mathrm{~S}_{6} \mathrm{P}_{2}\left\{\mathrm{OC}\left(\mathrm{CH}_{3}\right)_{2} \mathrm{C}\left(\mathrm{CH}_{3}\right)_{2} \mathrm{O}\right\}_{2}\right]$ & 1215 (1211.4) & $\begin{array}{c}26.71 \\
(26.75)\end{array}$ & $\begin{array}{c}2.17 \\
(2.21)\end{array}$ & $\begin{array}{l}2.30 \\
(2.31)\end{array}$ & $\begin{array}{c}15.82 \\
(15.85)\end{array}$ & $\begin{array}{c}15.02 \\
(15.06)\end{array}$ \\
\hline 24 & $\mathrm{Zr}_{2}\left(\mathrm{C}_{15} \mathrm{H}_{12} \mathrm{~N}_{2} \mathrm{OCl}\right)_{2}\left[\mathrm{~S}_{6} \mathrm{P}\right.$ & $1150(1152.4)$ & $\begin{array}{r}23.89 \\
(23.95) \\
\end{array}$ & $\begin{array}{l}2.06 \\
(2.08)\end{array}$ & $\begin{array}{l}2.42 \\
(2.43)\end{array}$ & $\begin{array}{c}16.63 \\
(16.66)\end{array}$ & $\begin{array}{r}15.80 \\
(15.83) \\
\end{array}$ \\
\hline
\end{tabular}

\subsection{IR Spectral Data}

The IR spectra shows bands of medium intensity in the region of $3382-3328 \mathrm{~cm}^{-1}$ due to $v[\mathrm{~N}-\mathrm{H}]$ stretching and vibrations and bands in the region of $1615-1580 \mathrm{~cm}^{-1}$ due to the $v[\mathrm{C}=\mathrm{N}]$ stretching vibration. In all the compounds the signal due to $v[\mathrm{C}=\mathrm{N}]$ stretching is found to be shifted to the lower wave number in comparison to the spectra of free pyrazolines (at $\sim 1654 \mathrm{~cm}^{-1}$ ), suggesting the involvement of imino nitrogen in coordination. The signal due to $\mathrm{v}(\mathrm{O}-\mathrm{H})$ stretching originally present at $\sim 3080 \mathrm{~cm}^{-1}$ in ligand is completely missing from the spectra of complexes. The bands observed in the region of 1042-1028 and 870$840 \mathrm{~cm}^{-1}$ have been assigned to $\mathrm{v}[(\mathrm{P})-\mathrm{O}-\mathrm{C}]$ and $v[\mathrm{P}-\mathrm{O}-$ (C)], respectively. The $v[\mathrm{P}=\mathrm{S}]$ mode may be characterized by the presence of bands in the region of $645-620 \mathrm{~cm}^{-1}$ indicating the bidentate nature of dithiophosphate ligands. The bands present in the region of $533-510 \mathrm{~cm}^{-1}$ may be ascribed to $v[\mathrm{P}-\mathrm{S}]$ stretching modes. Appearance of two new bands (in comparison to free ligand) in the region of 331-319 and 303-293 $\mathrm{cm}^{-1}$ indicates the formation of $v[\mathrm{Zr}-\mathrm{S}]$. Splitting of bands into two regions indicates that two types of sulphur are present in the molecule, one is terminal sulphur and another is bridging sulphur. The appearance of bands in the region of $392-367$ and $430-415 \mathrm{~cm}^{-1}$ has been assigned to $v[\mathrm{Zr}-\mathrm{N}]$ and $v[\mathrm{Zr}-\mathrm{O}]$ stretching vibration. The data is summarized in Table 2.

\section{2. ${ }^{1} \mathbf{H}$ N M R Spectra}

The ${ }^{1} \mathrm{H}$ NMR spectra of these new compounds have been recorded in $\mathrm{CDCl}_{3}$ exhibiting the characteristic signals. The aromatic protons of pyrazolines were observed as a complex pattern in the region $\delta=7.45-6.49 \mathrm{ppm}$. The peak due to hydroxyl protons (originally present at $\delta \sim 11.00 \mathrm{ppm}$ in free pyrazolines) is completely missing from the spectra of the complex suggesting the bonding through hydroxyl oxygen atom. The appearance of the peak at 5.32$4.75 \mathrm{ppm}$ as a broad singlet could be assigned to $\mathrm{N}-\mathrm{H}$ group (originally present at $\delta=5.4-5.0 \mathrm{ppm}$ ) indicating 
the non-involvement of $-\mathrm{NH}$ group. The skeletal protons of five membered rings are observed at $\delta=3.70$ $3.12 \mathrm{ppm}$ as a triplet and $\delta=2.95-1.02 \mathrm{ppm}$ could be assigned to $-\mathrm{CH}$ and $-\mathrm{CH}_{2}$ group. Band at 5.54-
$3.12 \mathrm{ppm}$ could be for $-\mathrm{OCH}_{2}$ and $-\mathrm{OCH}$ group and at $3.82-3.00 \mathrm{ppm}$ may be due to $-\mathrm{CH}$ group. The skeletal protons of phenyl ring are observed at 7.28-7.10 ppm.

Table 2

IR spectral data $\left(\mathrm{cm}^{-1}\right)$ for $\mathrm{Zr}_{2}\left(\mathrm{C}_{15} \mathrm{H}_{12} \mathrm{~N}_{2} \mathrm{OX}\right)_{2}\left[\mathrm{~S}_{6} \mathrm{P}_{2}(\mathrm{OR})_{4}\right]$

\begin{tabular}{|c|c|c|c|c|c|c|c|c|c|c|c|}
\hline No & $v[\mathrm{~N}-\mathrm{H}]$ & $v[\mathrm{C}=\mathrm{N}]$ & $v[\mathrm{C}-\mathrm{O}]$ & $v[(\mathrm{P})-\mathrm{O}-\mathrm{C}]$ & $v[\mathrm{P}-\mathrm{O}-(\mathrm{C})]$ & $v[\mathrm{P}=\mathrm{S}]$ & $v[\mathrm{P}-\mathrm{S}]$ & Ring vib & $v[\mathrm{Zr}-\mathrm{O}]$ & $v[\mathrm{Zr}-\mathrm{S}]$ & $v[\mathrm{Zr}-\mathrm{N}]$ \\
\hline 1 & 3371 & 1585 & - & 1031 & 867 & 623 & 511 & - & 423 & $\begin{array}{c}320 \\
295 \mathrm{~S}_{\mathrm{b}}\end{array}$ & 367 \\
\hline 2 & 3375 & 1591 & - & 1033 & 856 & 633 & 523 & - & 416 & $\begin{array}{r}324 \\
298 \mathrm{~S}_{\mathrm{b}} \\
\end{array}$ & 372 \\
\hline 3 & 3381 & 1609 & - & 1040 & 850 & 639 & 520 & 945 & 419 & $\begin{array}{c}321 \\
296 \mathrm{~S}_{\mathrm{b}} \\
\end{array}$ & 382 \\
\hline 4 & 3369 & 1614 & - & 1029 & 860 & 625 & 517 & 956 & 420 & $\begin{array}{r}329 \\
302 S_{b} \\
\end{array}$ & 390 \\
\hline 5 & 3370 & 1608 & - & 1033 & 850 & 636 & 519 & 963 & 431 & $\begin{array}{r}331 \\
295 \mathrm{~S}_{\mathrm{b}} \\
\end{array}$ & 369 \\
\hline 6 & 3379 & 1595 & - & 1041 & 853 & 641 & 521 & 954 & 428 & $\begin{array}{c}321 \\
298 S_{\mathrm{b}} \\
\end{array}$ & 374 \\
\hline 7 & 3380 & 1588 & - & 1029 & 845 & 638 & 523 & - & 419 & $\begin{array}{c}323 \\
301 S_{b} \\
\end{array}$ & 386 \\
\hline 8 & 3371 & 1592 & - & 1034 & 849 & 642 & 530 & - & 416 & $\begin{array}{c}325 \\
294 \mathrm{~S}_{\mathrm{b}} \\
\end{array}$ & 379 \\
\hline 9 & 3380 & 1603 & - & 1028 & 868 & 625 & 518 & 971 & 422 & $\begin{array}{c}328 \\
297 S_{b} \\
\end{array}$ & 381 \\
\hline 10 & 3379 & 1608 & - & 1030 & 854 & 631 & 529 & 963 & 426 & $\begin{array}{c}324 \\
298 S_{b} \\
\end{array}$ & 372 \\
\hline 11 & 3372 & 1583 & - & 1037 & 859 & 626 & 531 & 968 & 420 & $\begin{array}{r}327 \\
295 \mathrm{~S}_{\mathrm{b}} \\
\end{array}$ & 385 \\
\hline 12 & 3376 & 1599 & - & 1041 & 870 & 642 & 516 & 945 & 429 & $\begin{array}{c}319 \\
298 \mathrm{~S}_{\mathrm{b}} \\
\end{array}$ & 377 \\
\hline 13 & 3381 & 1600 & 1023 & 1038 & 842 & 645 & 518 & - & 421 & $\begin{array}{c}329 \\
303 \mathrm{~S}_{\mathrm{b}} \\
\end{array}$ & 389 \\
\hline 14 & 3368 & 1611 & 1017 & 1031 & 857 & 633 & 525 & - & 417 & $\begin{array}{c}320 \\
301 S_{b} \\
\end{array}$ & 390 \\
\hline 15 & 3379 & 1610 & 1020 & 1035 & 863 & 635 & 533 & 956 & 419 & $\begin{array}{r}330 \\
295 \mathrm{~S}_{\mathrm{b}} \\
\end{array}$ & 381 \\
\hline 16 & 3373 & 1590 & 1015 & 1042 & 870 & 638 & 511 & 963 & 431 & $\begin{array}{c}327 \\
297 \mathrm{~S}_{\mathrm{b}}\end{array}$ & 392 \\
\hline 17 & 3382 & 1598 & 1017 & 1029 & 860 & 631 & 516 & 961 & 427 & $\begin{array}{c}324 \\
294 S_{b}\end{array}$ & 387 \\
\hline 18 & 3370 & 1610 & 1022 & 1030 & 851 & 638 & 519 & 953 & 423 & $\begin{array}{c}329 \\
297 S_{b}\end{array}$ & 376 \\
\hline 19 & 3375 & 1615 & - & 1036 & 850 & 632 & 523 & - & 429 & $\begin{array}{c}321 \\
294 S_{b}\end{array}$ & 370 \\
\hline 20 & 3380 & 1589 & - & 1039 & 869 & 637 & 525 & - & 425 & $\begin{array}{c}320 \\
297 S_{b}\end{array}$ & 379 \\
\hline 21 & 3373 & 1593 & - & 1029 & 860 & 640 & 528 & 971 & 420 & $\begin{array}{c}327 \\
298 \mathrm{~S}_{\mathrm{b}}\end{array}$ & 383 \\
\hline 22 & 3378 & 1603 & - & 1040 & 852 & 632 & 521 & 964 & 416 & $\begin{array}{c}328 \\
303 \mathrm{~S}_{\mathrm{b}}\end{array}$ & 389 \\
\hline 23 & 3371 & 1598 & - & 1033 & 857 & 629 & 519 & 944 & 431 & $\begin{array}{c}322 \\
298 S_{b}\end{array}$ & 381 \\
\hline 24 & 3377 & 1611 & - & 1039 & 870 & 639 & 525 & 959 & 422 & $\begin{array}{c}325 \\
297 \mathrm{~S}_{\mathrm{b}}\end{array}$ & 290 \\
\hline
\end{tabular}




\section{3. ${ }^{13} \mathrm{C}$ N M R Spectra}

The proton decoupled ${ }^{13} \mathrm{C}$ NMR spectra show the presence of all important signals with reference to dithiophosphates and pyrazolines. The signals observed in the region $\delta=135.20-123.30 \mathrm{ppm}$ as a complex pattern could be assigned to aromatic carbon atoms ${ }^{233}$. The signal observed at $\delta=167.75-162.35 \mathrm{ppm}$ due to imino carbon of $\mathrm{C}=\mathrm{N}$ has shifted downfield in comparison to spectra of free pyrazolines $(\delta=143.50-142.80 \mathrm{ppm})$, suggesting the involvement of imino nitrogen in coordination. The peaks due to $-\mathrm{CH}$ and $-\mathrm{CH}_{2}$ are observed at $46.15-41.08 \mathrm{ppm}$. The peaks due to $-\mathrm{OC}$ and $-\mathrm{OCH}$ group of dithiophosphates are observed at 90.15-91.26 and 77.35-75.29 ppm, respectively.

\section{4. ${ }^{31}$ P N M R Data}

In ${ }^{31} \mathrm{P}$ NMR spectra of these compounds only one signal has been observed for the phosphorus atom. The ${ }^{31} \mathrm{P}$ NMR signals of $\mathrm{Zr}$ dichlorodithiocompounds are observed at $\delta=90 \mathrm{ppm}$, while the ${ }^{31} \mathrm{P}$ NMR signals of synthesized $\mathrm{Zr}$ compounds are obtained at $\delta=107.7-93 \mathrm{ppm}$. The downfield shifting of the signal at $\sim 15.0 \mathrm{ppm}$ due to dithiophosphate phosphorus atom confirms the bidentate nature of the ligands. Despite the presence of two phosphorus atoms, only one signal is obtained, indicating similar environment for both phosphorus atoms. The NMR $\left({ }^{1} \mathrm{H},{ }^{13} \mathrm{C}\right.$ and $\left.{ }^{31} \mathrm{P}\right)$ data are summarized in Table 3.

Table 3

NMR data for $\mathrm{Zr}_{2}\left(\mathrm{C}_{15} \mathrm{H}_{12} \mathrm{~N}_{2} \mathrm{OX}\right)_{2}\left[\mathrm{~S}_{6} \mathrm{P}_{2}(\mathrm{OR})_{4}\right]$

\begin{tabular}{|c|c|c|c|c|}
\hline \multirow{2}{*}{ No. } & \multirow{2}{*}{ Compound } & \multicolumn{3}{|c|}{ Chemical shift $(\delta), \mathrm{ppm}$} \\
\hline & & ${ }^{\mathrm{T}} \mathrm{H}$ NMR & ${ }^{13} \mathrm{C} \mathrm{NMR}$ & ${ }^{31} \mathrm{P}$ NMR \\
\hline 1 & 2 & 3 & 4 & 5 \\
\hline 1 & $\mathrm{Zr}_{2}\left(\mathrm{C}_{15} \mathrm{H}_{12} \mathrm{~N}_{2} \mathrm{OH}\right)_{2}\left[\mathrm{~S}_{6} \mathrm{P}_{2}\left(\mathrm{OCH}_{2} \mathrm{CH}_{2} \mathrm{CH}_{3}\right)_{4}\right]$ & $\begin{array}{c}7.45-6.80(\mathrm{~m}, 18 \mathrm{H}, \mathrm{Ar}- \\
\mathrm{H}) \\
0.96\left(\mathrm{t}, 12 \mathrm{H},-\mathrm{CH}_{3}\right) \\
5.17\left(\mathrm{~m}, 8 \mathrm{H},-\mathrm{OCH}_{2}\right) \\
1.70\left(\mathrm{~m}, 8 \mathrm{H},-\mathrm{CH}_{2}\right) \\
5.21\left(\mathrm{~s}, 2 \mathrm{H},-\mathrm{NH}^{-}\right) \\
3.19(\mathrm{t}, 2 \mathrm{H},-\mathrm{CH}) \\
2.19\left(\mathrm{~d}, 4 \mathrm{H},-\mathrm{CH}_{2}\right)\end{array}$ & - & 103.00 \\
\hline 2 & $\mathrm{Zr}_{2}\left(\mathrm{C}_{15} \mathrm{H}_{12} \mathrm{~N}_{2} \mathrm{OH}\right)_{2}\left[\mathrm{~S}_{6} \mathrm{P}_{2}\left(\mathrm{OC}_{6} \mathrm{H}_{5}\right)_{4}\right]$ & $\begin{array}{c}7.74-6.95(\mathrm{~m}, 18 \mathrm{H}, \mathrm{Ar}- \\
\mathrm{H}) \\
7.25\left(\mathrm{~s}, 2 \mathrm{H},-\mathrm{C}_{6} \mathrm{H}_{5}\right) \\
4.82(\mathrm{~s}, 2 \mathrm{H},-\mathrm{NH}) \\
3.21(\mathrm{t}, 2 \mathrm{H},-\mathrm{CH}) \\
2.54\left(\mathrm{~d}, 4 \mathrm{H}-\mathrm{CH}_{2}\right)\end{array}$ & - & 93.00 \\
\hline 3 & $\mathrm{Zr}_{2}\left(\mathrm{C}_{15} \mathrm{H}_{12} \mathrm{~N}_{2} \mathrm{OH}\right)_{2}\left[\mathrm{~S}_{6} \mathrm{P}_{2}\left\{\mathrm{OC}\left(\mathrm{CH}_{3}\right)_{2} \mathrm{CH}_{2} \mathrm{CH}\left(\mathrm{CH}_{3}\right) \mathrm{O}\right\}_{2}\right]$ & $\begin{array}{c}7.64-6.90(\mathrm{~m}, 18 \mathrm{H}, \mathrm{Ar}- \\
\mathrm{H}) \\
2.97-2.60(\mathrm{~m}, 22 \mathrm{H},- \\
\left.\mathrm{CH}_{3}, \mathrm{CH}_{2}\right) \\
5.54(\mathrm{~m}, 2 \mathrm{H},-\mathrm{OCH}) \\
4.64(\mathrm{~s}, 2 \mathrm{H},-\mathrm{NH}) \\
3.52(\mathrm{t}, 2 \mathrm{H},-\mathrm{CH}) \\
2.18\left(\mathrm{~d}, 4 \mathrm{H},-\mathrm{CH}_{2}\right)\end{array}$ & $\begin{array}{c}\text { 91.45(-OC, dtp) } \\
24.45\left(-\mathrm{CH}_{3}, \mathrm{dtp}\right) \\
77.01(-\mathrm{OCH}, \mathrm{dtp}) \\
26.32\left(-\mathrm{CH}_{2}, \mathrm{dtp}\right) \\
136.72(\mathrm{Ar}-\mathrm{C}) \\
167.75(\mathrm{C}=\mathrm{N}) \\
43.02(-\mathrm{CH}) \\
25.83\left(-\mathrm{CH}_{2}\right)\end{array}$ & 102.20 \\
\hline 4 & $\mathrm{Zr}_{2}\left(\mathrm{C}_{15} \mathrm{H}_{12} \mathrm{~N}_{2} \mathrm{OH}\right)_{2}\left[\mathrm{~S}_{6} \mathrm{P}_{2}\left\{\mathrm{OCH}_{2} \mathrm{C}\left(\mathrm{CH}_{3}\right)_{2} \mathrm{CH}_{2} \mathrm{O}\right\}_{2}\right]$ & $\begin{array}{c}7.64-6.83(\mathrm{~m}, 18 \mathrm{H}, \mathrm{Ar}- \\
\mathrm{H}) \\
0.97\left(\mathrm{~s}, 12 \mathrm{H},-\mathrm{CH}_{3}\right) \\
5.17\left(\mathrm{~d}, 8 \mathrm{H},-\mathrm{OCH}_{2}-\right) \\
4.76\left(\mathrm{~s}, 2 \mathrm{H}, \mathrm{NH}^{-}\right) \\
3.43(\mathrm{t}, 2 \mathrm{H},-\mathrm{CH}) \\
2.19\left(\mathrm{~d}, 4 \mathrm{H},-\mathrm{CH}_{2}\right)\end{array}$ & $\begin{array}{c}21.87\left(\mathrm{CH}_{3,} \mathrm{dtp}\right) \\
31.82(\mathrm{qC}, \mathrm{dtp}) \\
76.32\left(-\mathrm{OCH}_{2}, \mathrm{dtp}\right) \\
136.83(\mathrm{Ar}-\mathrm{C}) \\
165.51(\mathrm{C}=\mathrm{N}) \\
42.92(\mathrm{CH}) \\
27.63\left(\mathrm{CH}_{2}\right)\end{array}$ & 97.80 \\
\hline 5 & $\left.\mathrm{Zr}_{2}\left(\mathrm{C}_{15} \mathrm{H}_{12} \mathrm{~N}_{2} \mathrm{OH}\right)_{2}\left[\mathrm{~S}_{6} \mathrm{P}_{2 i} \mathrm{OC}\left(\mathrm{CH}_{3}\right)_{2} \mathrm{C}_{\left(\mathrm{CH}_{3}\right)_{2} \mathrm{O}}\right\}_{2}\right]$ & $\begin{array}{c}1.12\left(\mathrm{~s}, 24 \mathrm{H},-\mathrm{CH}_{3}\right) \\
\text { 7.69-6.78(m, 18H, ArH }) \\
4.75(\mathrm{~s}, 2 \mathrm{H},-\mathrm{NH}) \\
3.02(\mathrm{t}, 2 \mathrm{H},-\mathrm{CH}) \\
2.05\left(\mathrm{~d}, 4 \mathrm{H},-\mathrm{CH}_{2}\right)\end{array}$ & $\begin{array}{c}23.95\left(\mathrm{CH}_{3}, \mathrm{dtp}\right) \\
90.52(\mathrm{OC}, \mathrm{dtp}) \\
136.61-122.14(\mathrm{Ar}- \\
\mathrm{C}) \\
165.24(\mathrm{C}=\mathrm{N}) \\
42.18(\mathrm{CH}) \\
27.78\left(\mathrm{CH}_{2}\right)\end{array}$ & 104.50 \\
\hline
\end{tabular}


Table 3 (continued)

\begin{tabular}{|c|c|c|c|c|}
\hline 1 & 2 & 3 & 4 & 5 \\
\hline 6 & 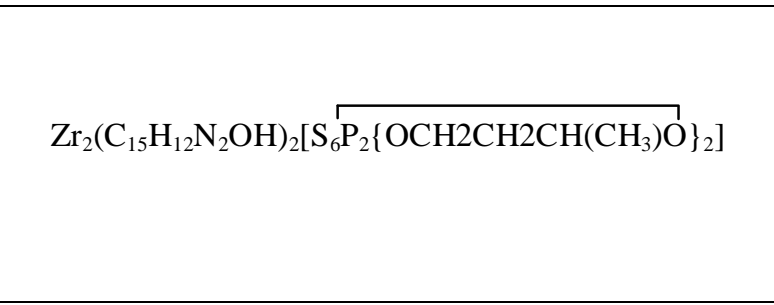 & $\begin{array}{c}2.46-1.05\left(\mathrm{~m}, 10 \mathrm{H},-\mathrm{CH}_{3},\right. \\
\left.\mathrm{CH}_{2}\right) \\
4.42-3.15\left(\mathrm{~m}, 6 \mathrm{H},-\mathrm{OCH}_{2},\right. \\
\mathrm{OCH}) \\
7.80-6.92(\mathrm{~m}, 18 \mathrm{H}, \mathrm{ArH}) \\
4.96(\mathrm{~s}, 2 \mathrm{H},-\mathrm{NH}) \\
3.27(\mathrm{t}, 2 \mathrm{H},-\mathrm{CH}) \\
2.21\left(\mathrm{~d}, 4 \mathrm{H},-\mathrm{CH}_{2}\right) \\
\end{array}$ & $\begin{array}{c}23.81\left(\mathrm{CH}_{3}, \mathrm{dtp}\right) \\
76.12\left(-\mathrm{OCH}, \mathrm{OCH}_{2,}\right. \\
\mathrm{dtp}) \\
135.25-123.24(\mathrm{Ar}-\mathrm{C}) \\
163.21(\mathrm{C}=\mathrm{N}) \\
43.05(\mathrm{CH}) \\
27.51\left(\mathrm{CH}_{2}\right)\end{array}$ & 103.90 \\
\hline 7 & $\mathrm{Zr}_{2}\left(\mathrm{C}_{15} \mathrm{H}_{12} \mathrm{~N}_{2} \mathrm{OCH}_{3}\right)_{2}\left[\mathrm{~S}_{6} \mathrm{P}_{2}\left(\mathrm{OCH}_{2} \mathrm{CH}_{2} \mathrm{CH}_{3}\right)_{4}\right]$ & $\begin{array}{c}7.73-6.72(\mathrm{~m}, 16 \mathrm{H}, \mathrm{Ar}-\mathrm{H}) \\
0.97\left(\mathrm{t}, 12 \mathrm{H},-\mathrm{CH}_{3}\right) \\
5.07\left(\mathrm{~m}, 8 \mathrm{H},-\mathrm{OCH}_{2}-\right) \\
1.24\left(\mathrm{~m}, 8 \mathrm{H},-\mathrm{CH}_{2}\right) \\
4.97(\mathrm{~s}, 2 \mathrm{H}, \mathrm{NH}) \\
3.22(\mathrm{t}, 2 \mathrm{H},-\mathrm{CH}) \\
2.15\left(\mathrm{~d}, 4 \mathrm{H},-\mathrm{CH}_{2}\right) \\
1.02\left(\mathrm{~s}, 6 \mathrm{H}, \mathrm{CH}_{3}\right) \\
\end{array}$ & - & 93.60 \\
\hline 8 & $\mathrm{Zr}_{2}\left(\mathrm{C}_{15} \mathrm{H}_{12} \mathrm{~N}_{2} \mathrm{OCH}_{3}\right)_{2}\left[\mathrm{~S}_{6} \mathrm{P}_{2}\left(\mathrm{OC}_{6} \mathrm{H}_{5}\right)_{4}\right]$ & $\begin{array}{c}7.67-6.57(\mathrm{~m}, 16 \mathrm{H}, \mathrm{Ar}-\mathrm{H}) \\
7.26\left(\mathrm{~s}, 20 \mathrm{H},-\mathrm{C}_{6} \mathrm{H}_{5}\right) \\
5.02(\mathrm{~s}, 2 \mathrm{H},-\mathrm{NH}) \\
3.20(\mathrm{t}, 2 \mathrm{H},-\mathrm{CH}) \\
2.27\left(\mathrm{~d}, 4 \mathrm{H}-\mathrm{CH}_{2}\right) \\
0.98\left(\mathrm{~s}, 6 \mathrm{H},-\mathrm{CH}_{3}\right)\end{array}$ & - & 99.00 \\
\hline 9 & $\mathrm{Zr}_{2}\left(\mathrm{C}_{15} \mathrm{H}_{12} \mathrm{~N}_{2} \mathrm{OCH}_{3}\right)_{2}\left[\mathrm{~S}_{6} \mathrm{P}_{2}\left\{\mathrm{OC}\left(\mathrm{CH}_{3}\right)_{2} \mathrm{CH}_{2} \mathrm{CH}\left(\mathrm{CH}_{3}\right) \mathrm{O}\right\}_{2}\right]$ & $\begin{array}{c}7.56-6.71(\mathrm{~m}, 16 \mathrm{H}, \mathrm{Ar}- \\
\mathrm{H}) \\
2.47-2.17\left(\mathrm{~m}, 22 \mathrm{H},-\mathrm{CH}_{3},\right. \\
\left.\mathrm{CH}_{2}\right) \\
4.96-4.58(\mathrm{~m}, 2 \mathrm{H},-\mathrm{OCH}) \\
5.10(\mathrm{~s}, 2 \mathrm{H},-\mathrm{NH}) \\
3.21(\mathrm{t}, 2 \mathrm{H},-\mathrm{CH}) \\
2.03\left(\mathrm{~d}, 4 \mathrm{H}, \mathrm{CH}_{2}\right) \\
1.05\left(\mathrm{~s}, 6 \mathrm{H},-\mathrm{CH}_{3}\right)\end{array}$ & $\begin{array}{c}91.26(-\mathrm{OC}, \mathrm{dtp}) \\
25.81\left(-\mathrm{CH}_{3}, \mathrm{dtp}\right) \\
76.75(-\mathrm{OCH}, \mathrm{dtp}) \\
23.34\left(-\mathrm{CH}_{2}, \mathrm{dtp}\right) \\
135.75(\mathrm{Ar}-\mathrm{C}) \\
163.72(\mathrm{C}=\mathrm{N}) \\
42.31(-\mathrm{CH}) \\
23.91\left(-\mathrm{CH}_{2}\right) \\
13.51\left(-\mathrm{CH}_{3}\right) \\
\end{array}$ & 105.00 \\
\hline 10 & $\mathrm{Zr}_{2}\left(\mathrm{C}_{15} \mathrm{H}_{12} \mathrm{~N}_{2} \mathrm{OCH}_{3}\right)_{2}\left[\mathrm{~S}_{6} \mathrm{P}_{2}\left\{\mathrm{OCH}_{2} \mathrm{C}\left(\mathrm{CH}_{3}\right)_{2} \mathrm{CH}_{2} \mathrm{O}\right\}_{2}\right]$ & $\begin{array}{c}7.71-6.49(\mathrm{~m}, 16 \mathrm{H}, \mathrm{Ar}-\mathrm{H}) \\
0.97\left(\mathrm{~s}, 12 \mathrm{H},-\mathrm{CH}_{3}\right) \\
4.23\left(\mathrm{~d}, 8 \mathrm{H},-\mathrm{OCH}_{2}-\right) \\
5.17(\mathrm{~s}, 4 \mathrm{H}, \mathrm{NH}) \\
3.71(\mathrm{t}, 2 \mathrm{H},-\mathrm{CH}) \\
2.03\left(\mathrm{~d}, 8 \mathrm{H},-\mathrm{CH}_{2}\right) \\
0.95\left(\mathrm{~s}, 6 \mathrm{H},-\mathrm{CH}_{3}\right)\end{array}$ & $\begin{array}{c}22.30\left(\mathrm{CH}_{3}, \mathrm{dtp}\right) \\
31.31(\mathrm{q} \mathrm{C}, \mathrm{dtp}) \\
75.29\left(-\mathrm{OCH} \mathrm{H}_{2,} \mathrm{dtp}\right) \\
136.39(\mathrm{Ar}-\mathrm{C}) \\
165.75(\mathrm{C}=\mathrm{N}) \\
43.17(\mathrm{CH}) \\
26.37\left(\mathrm{CH}_{2}\right) \\
13.47\left(-\mathrm{CH}_{3}\right) \\
\end{array}$ & 104.60 \\
\hline 11 & $\mathrm{Zr}_{2}\left(\mathrm{C}_{15} \mathrm{H}_{12} \mathrm{~N}_{2} \mathrm{OCH}_{3}\right)_{2}\left[\mathrm{~S}_{6} \mathrm{P}_{2}\left\{\mathrm{OC}\left(\mathrm{CH}_{3}\right)_{2} \mathrm{C}\left(\mathrm{CH}_{3}\right)_{2} \mathrm{O}\right\}_{2}\right]$ & $\begin{array}{c}1.32\left(\mathrm{~s}, 24 \mathrm{H},-\mathrm{CH}_{3}\right) \\
7.57-6.68(\mathrm{~m}, 16 \mathrm{H}, \mathrm{ArH}) \\
4.83(\mathrm{~s}, 2 \mathrm{H},-\mathrm{NH}) \\
3.35(\mathrm{t}, 2 \mathrm{H},-\mathrm{CH}) \\
2.15\left(\mathrm{~d}, 4 \mathrm{H},-\mathrm{CH}_{2}\right) \\
0.97\left(\mathrm{~s}, 6 \mathrm{H},-\mathrm{CH}_{3}\right)\end{array}$ & $\begin{array}{c}26.12\left(\mathrm{CH}_{3}, \mathrm{dtp}\right) \\
91.95(\mathrm{OC}, \mathrm{dtp}) \\
136.36-123.42(\mathrm{Ar}-\mathrm{C}) \\
166.85(\mathrm{C}=\mathrm{N}) \\
46.15(\mathrm{CH}) \\
25.15\left(\mathrm{CH}_{2}\right) \\
13.36\left(-\mathrm{CH}_{3}\right)\end{array}$ & 105.95 \\
\hline 12 & $\mathrm{Zr}_{2}\left(\mathrm{C}_{15} \mathrm{H}_{12} \mathrm{~N}_{2} \mathrm{OCH}_{3}\right)_{2}\left[\mathrm{~S}_{6} \mathrm{P}_{2}\left\{\mathrm{OCH}_{2} \mathrm{CH}_{2} \mathrm{CH}\left(\mathrm{CH}_{3}\right) \mathrm{O}\right\}_{2}\right]$ & $\begin{array}{c}2.05-1.10\left(\mathrm{~m}, 10 \mathrm{H},-\mathrm{CH}_{3},\right. \\
\left.\mathrm{CH}_{2}\right) \\
4.56-3.15\left(\mathrm{~m}, 6 \mathrm{H},-\mathrm{OCH}_{2},\right. \\
\mathrm{OCH}) \\
7.60-6.75(\mathrm{~m}, 16 \mathrm{H}, \mathrm{ArH}) \\
5.15(\mathrm{~s}, 2 \mathrm{H},-\mathrm{NH}) \\
2.76(\mathrm{t}, 2 \mathrm{H},-\mathrm{CH}) \\
1.97\left(\mathrm{~d}, 4 \mathrm{H},-\mathrm{CH}_{2}\right) \\
1.02\left(\mathrm{~s}, 6 \mathrm{H},-\mathrm{CH}_{3}\right)\end{array}$ & $\begin{array}{c}24.29\left(\mathrm{CH}_{3}, \mathrm{dtp}\right) \\
76.54\left(-\mathrm{OCH}, \mathrm{OCH}_{2,}\right. \\
\mathrm{dtp}) \\
136.29-123.65(\mathrm{Ar}-\mathrm{C}) \\
165.25(\mathrm{C}=\mathrm{N}) \\
42.92(\mathrm{CH}) \\
27.29\left(\mathrm{CH}_{2}\right) \\
13.54\left(-\mathrm{CH}_{3}\right)\end{array}$ & 101.76 \\
\hline
\end{tabular}


Synthesis and Characterization of M ixed Ligand Complexes of Zirconium(IV) with Sulphur...

Table 3 (continued)

\begin{tabular}{|c|c|c|c|c|}
\hline 1 & 2 & 3 & 4 & 5 \\
\hline 13 & $\mathrm{Zr}_{2}\left(\mathrm{C}_{15} \mathrm{H}_{12} \mathrm{~N}_{2} \mathrm{O}_{2} \mathrm{CH}_{3}\right)_{2}\left[\mathrm{~S}_{6} \mathrm{P}_{2}\left(\mathrm{OCH}_{2} \mathrm{CH}_{2} \mathrm{CH}_{3}\right)_{4}\right]$ & $\begin{array}{c}7.82-6.95(\mathrm{~m}, 16 \mathrm{H}, \mathrm{Ar}-\mathrm{H}) \\
1.05\left(\mathrm{t}, 12 \mathrm{H},-\mathrm{CH}_{3}\right) \\
5.10\left(\mathrm{~m}, 8 \mathrm{H},-\mathrm{OCH}_{2}-\right) \\
1.45\left(\mathrm{~m}, 8 \mathrm{H},-\mathrm{CH}_{2}\right) \\
5.07(\mathrm{~s}, 2 \mathrm{H}, \mathrm{NH}) \\
3.76(\mathrm{t}, 2 \mathrm{H},-\mathrm{CH}) \\
2.19\left(\mathrm{~d}, 4 \mathrm{H},-\mathrm{CH}_{2}\right) \\
3.46\left(\mathrm{~s}, 6 \mathrm{H}, \mathrm{OCH}_{3}\right)\end{array}$ & - & 93.50 \\
\hline 14 & $\mathrm{Zr}_{2}\left(\mathrm{C}_{15} \mathrm{H}_{12} \mathrm{~N}_{2} \mathrm{O}_{2} \mathrm{CH}_{3}\right)_{2}\left[\mathrm{~S}_{6} \mathrm{P}_{2}\left(\mathrm{OC}_{6} \mathrm{H}_{5}\right)_{4}\right]$ & $\begin{array}{c}7.85-6.90(\mathrm{~m}, 16 \mathrm{H}, \mathrm{Ar}-\mathrm{H}) \\
7.28\left(\mathrm{~s}, 20 \mathrm{H},-\mathrm{C}_{6} \mathrm{H}_{5}\right) \\
4.95(\mathrm{~s}, 2 \mathrm{H},-\mathrm{NH}) \\
3.09(\mathrm{t}, 2 \mathrm{H},-\mathrm{CH}) \\
2.02\left(\mathrm{~d}, 4 \mathrm{H},-\mathrm{CH}_{2}\right) \\
3.39\left(\mathrm{~s}, 6 \mathrm{H}, \mathrm{OCH}_{3}\right) \\
\end{array}$ & - & 98.90 \\
\hline 15 & 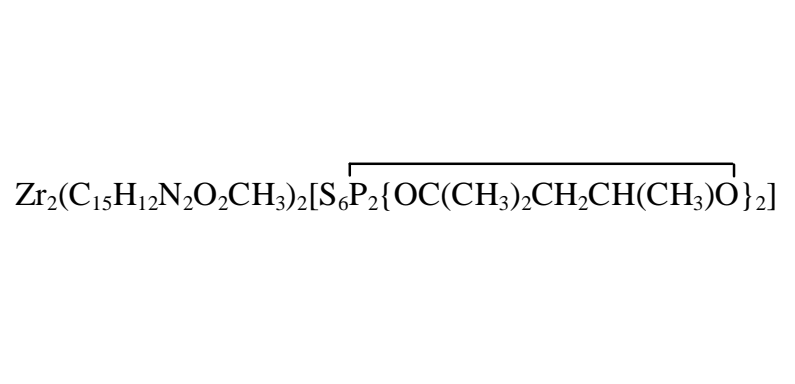 & $\begin{array}{c}7.75-6.81(\mathrm{~m}, 16 \mathrm{H}, \mathrm{Ar}-\mathrm{H}) \\
2.30-1.95\left(\mathrm{~m}, 22 \mathrm{H},-\mathrm{CH}_{3},\right. \\
\left.\mathrm{CH}_{2}\right) \\
4.56-4.20(\mathrm{~m}, 2 \mathrm{H},-\mathrm{OCH}) \\
5.02(\mathrm{~s}, 2 \mathrm{H},-\mathrm{NH}) \\
3.10(\mathrm{t}, 2 \mathrm{H},-\mathrm{CH}) \\
2.17\left(\mathrm{~d}, 4 \mathrm{H}, \mathrm{CH}_{2}\right) \\
3.32\left(\mathrm{~s}, 6 \mathrm{H}, \mathrm{OCH}_{3}\right)\end{array}$ & $\begin{array}{c}91.95(-\mathrm{OC}, \mathrm{dtp}) \\
24.75\left(-\mathrm{CH}_{3}, \mathrm{dtp}\right) \\
76.43(-\mathrm{OCH}, \mathrm{dtp}) \\
23.15\left(-\mathrm{CH}_{2}, \mathrm{dtp}\right) \\
136.34(\mathrm{Ar}-\mathrm{C}) \\
167.10(\mathrm{C}=\mathrm{N}) \\
43.12(-\mathrm{CH}) \\
24.19\left(-\mathrm{CH}_{2}\right) \\
57.49\left(-\mathrm{OCH}_{3}\right)\end{array}$ & 100.60 \\
\hline 16 & $\mathrm{Zr}_{2}\left(\mathrm{C}_{15} \mathrm{H}_{12} \mathrm{~N}_{2} \mathrm{O}_{2} \mathrm{CH}_{3}\right)_{2}\left[\mathrm{~S}_{6} \mathrm{P}_{2}\left\{\mathrm{OCH}_{2} \mathrm{C}\left(\mathrm{CH}_{3}\right)_{2} \mathrm{CH}_{2} \mathrm{O}\right\}_{2}\right]$ & $\begin{array}{c}7.50-6.85(\mathrm{~m}, 16 \mathrm{H}, \mathrm{Ar}-\mathrm{H}) \\
1.21\left(\mathrm{~s}, 12 \mathrm{H},-\mathrm{CH}_{3}\right) \\
4.12\left(\mathrm{~d}, 8 \mathrm{H},-\mathrm{OCH}_{2}-\right) \\
5.32(\mathrm{~s}, 2 \mathrm{H}, \mathrm{NH}) \\
3.12(\mathrm{t}, 2 \mathrm{H},-\mathrm{CH}) \\
2.32\left(\mathrm{~d}, 4 \mathrm{H},-\mathrm{CH}_{2}\right) \\
3.45\left(\mathrm{~s}, 6 \mathrm{H}, \mathrm{OCH}_{3}\right)\end{array}$ & \begin{tabular}{|c|}
$22.75\left(\mathrm{CH}_{3}, \mathrm{dtp}\right)$ \\
$32.32(\mathrm{q} \mathrm{C}, \mathrm{dtp})$ \\
$75.85\left(\mathrm{~d},-\mathrm{OCH} \mathrm{CH}_{2,} \mathrm{dtp}\right)$ \\
$137.30(\mathrm{Ar}-\mathrm{C})$ \\
$167.26(\mathrm{C}=\mathrm{N})$ \\
$42.84(\mathrm{CH})$ \\
$27.16\left(\mathrm{CH}_{2}\right)$ \\
$57.24\left(-\mathrm{OCH}_{3}\right)$ \\
\end{tabular} & 97.95 \\
\hline 17 & 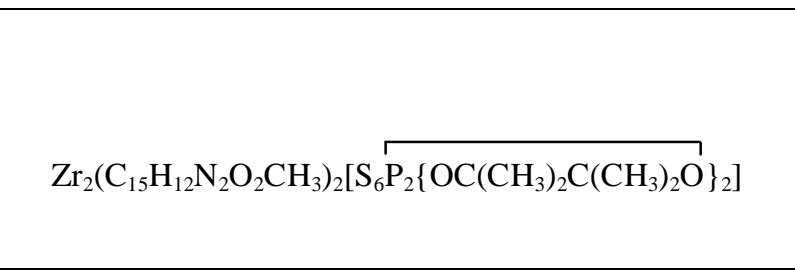 & $\begin{array}{c}1.27\left(\mathrm{~s}, 24 \mathrm{H},-\mathrm{CH}_{3}\right) \\
7.76-6.93(\mathrm{~m}, 16 \mathrm{H}, \mathrm{ArH}) \\
5.17(\mathrm{~s}, 2 \mathrm{H},-\mathrm{NH}) \\
3.21(\mathrm{t}, 2 \mathrm{H},-\mathrm{CH}) \\
2.03\left(\mathrm{~d}, 4 \mathrm{H},-\mathrm{CH}_{2}\right) \\
3.64\left(\mathrm{~s}, 6 \mathrm{H}, \mathrm{OCH}_{3}\right)\end{array}$ & \begin{tabular}{|c|}
$23.12\left(\mathrm{CH}_{3}, \mathrm{dtp}\right)$ \\
$90.15(\mathrm{OC}, \mathrm{dtp})$ \\
$136.12-123.45(\mathrm{Ar}-\mathrm{C})$ \\
$165.56(\mathrm{C}=\mathrm{N})$ \\
$41.08(\mathrm{CH})$ \\
$27.09\left(\mathrm{CH}_{2}\right)$ \\
$57.20\left(-\mathrm{OCH}_{3}\right)$ \\
\end{tabular} & 107.70 \\
\hline 18 & $\mathrm{Zr}_{2}\left(\mathrm{C}_{15} \mathrm{H}_{12} \mathrm{~N}_{2} \mathrm{O}_{2} \mathrm{CH}_{3}\right)_{2}\left[\mathrm{~S}_{6} \mathrm{P}_{2}\left\{\mathrm{OCH}_{2} \mathrm{CH}_{2} \mathrm{CH}\left(\mathrm{CH}_{3}\right) \mathrm{O}\right\}_{2}\right]$ & $\begin{array}{c}2.75-1.32\left(\mathrm{~m}, 10 \mathrm{H},-\mathrm{CH}_{3},\right. \\
\left.\mathrm{CH}_{2}\right) \\
4.09-3.76\left(\mathrm{~m}, 6 \mathrm{H},-\mathrm{OCH}_{2},\right. \\
\mathrm{OCH}) \\
7.79-6.65(\mathrm{~m}, 16 \mathrm{H}, \mathrm{ArH}) \\
5.21(\mathrm{~s}, 2 \mathrm{H},-\mathrm{NH}) \\
3.34(\mathrm{t}, 2 \mathrm{H},-\mathrm{CH}) \\
2.19\left(\mathrm{~d}, 4 \mathrm{H},-\mathrm{CH}_{2}\right) \\
3.60\left(\mathrm{~s}, 6 \mathrm{H}, \mathrm{OCH}_{3}\right)\end{array}$ & \begin{tabular}{|c|}
$22.28\left(\mathrm{CH}_{3}, \mathrm{dtp}\right)$ \\
$76.10\left(-\mathrm{OCH}, \mathrm{OCH}_{2,}\right.$ \\
dtp) \\
$136.36-123.35(\mathrm{Ar}-\mathrm{C})$ \\
$162.35(\mathrm{C}=\mathrm{N})$ \\
$42.34(\mathrm{CH})$ \\
$27.87\left(\mathrm{CH}_{2}\right)$ \\
$56.89\left(-\mathrm{OCH}_{3}\right)$
\end{tabular} & 103.70 \\
\hline 19 & $\mathrm{Zr}_{2}\left(\mathrm{C}_{15} \mathrm{H}_{12} \mathrm{~N}_{2} \mathrm{OCl}\right)_{2}\left[\mathrm{~S}_{6} \mathrm{P}_{2}\left(\mathrm{OCH}_{2} \mathrm{CH}_{2} \mathrm{CH}_{3}\right)_{4}\right]$ & $\begin{array}{c}7.75-6.56(\mathrm{~m}, 16 \mathrm{H}, \mathrm{Ar}-\mathrm{H}) \\
1.03\left(\mathrm{t}, 12 \mathrm{H},-\mathrm{CH}_{3}\right) \\
5.34\left(\mathrm{~m}, 8 \mathrm{H},-\mathrm{OCH}_{2}-\right) \\
1.60\left(\mathrm{~m}, 8 \mathrm{H},-\mathrm{CH}_{2}\right) \\
5.21(\mathrm{~s}, 2 \mathrm{H}, \mathrm{NH}) \\
3.45(\mathrm{t}, 2 \mathrm{H},-\mathrm{CH}) \\
2.15\left(\mathrm{~d}, 4 \mathrm{H},-\mathrm{CH}_{2}\right)\end{array}$ & - & 93.30 \\
\hline
\end{tabular}


Table 3 (continued)

\begin{tabular}{|c|c|c|c|c|}
\hline 1 & 2 & 3 & 4 & 5 \\
\hline 20 & $\mathrm{Zr}_{2}\left(\mathrm{C}_{15} \mathrm{H}_{12} \mathrm{~N}_{2} \mathrm{OCl}\right)_{2}\left[\mathrm{~S}_{6} \mathrm{P}_{2}\left(\mathrm{OC}_{6} \mathrm{H}_{5}\right)_{4}\right]$ & $\begin{array}{c}\text { 7.85-6.70(m,16H Ar-H }) \\
7.10\left(\mathrm{~s}, 20 \mathrm{H},-\mathrm{C}_{6} \mathrm{H}_{5}\right) \\
4.97(\mathrm{~s}, 2 \mathrm{H},-\mathrm{NH}) \\
3.10(\mathrm{t}, 2 \mathrm{H},-\mathrm{CH}) \\
2.15\left(\mathrm{~d}, 4 \mathrm{H}-\mathrm{CH}_{2}\right) \\
\end{array}$ & - & 105.50 \\
\hline 21 & 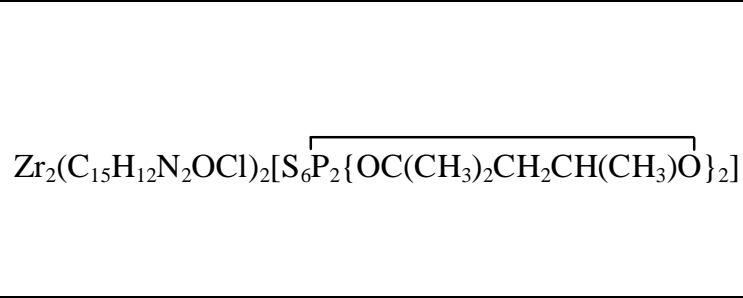 & $\begin{array}{c}7.65-6.80(\mathrm{~m}, 16 \mathrm{H}, \mathrm{Ar}-\mathrm{H}) \\
2.40-1.59\left(\mathrm{~m}, 22 \mathrm{H},-\mathrm{CH}_{3},\right. \\
\left.\mathrm{CH}_{2}\right) \\
4.70-4.15(\mathrm{~m}, 2 \mathrm{H},-\mathrm{OCH}) \\
5.07(\mathrm{~s}, 2 \mathrm{H},-\mathrm{NH}) \\
3.12(\mathrm{t}, 2 \mathrm{H},-\mathrm{CH}) \\
2.21\left(\mathrm{~d}, 4 \mathrm{H}, \mathrm{CH}_{2}\right)\end{array}$ & $\begin{array}{c}91.83(-\mathrm{OC}, \mathrm{dtp}) \\
23.72\left(-\mathrm{CH}_{3}, \mathrm{dtp}\right) \\
77.35(-\mathrm{OCH}, \mathrm{dtp}) \\
24.62\left(-\mathrm{CH}_{2}, \mathrm{dtp}\right) \\
136.73(\mathrm{Ar}-\mathrm{C}) \\
167.48(\mathrm{C}=\mathrm{N}) \\
42.73(-\mathrm{CH}) \\
27.32\left(-\mathrm{CH}_{2}\right) \\
\end{array}$ & 101.50 \\
\hline 22 & $\mathrm{Zr}_{2}\left(\mathrm{C}_{15} \mathrm{H}_{12} \mathrm{~N}_{2} \mathrm{OCl}\right)_{2}\left[\mathrm{~S}_{6} \mathrm{P}_{2}\left\{\mathrm{OCH}_{2} \mathrm{C}\left(\mathrm{CH}_{3}\right)_{2} \mathrm{CH}_{2} \mathrm{O}\right\}_{2}\right]$ & $\begin{array}{c}7.75-6.65(\mathrm{~m}, 16 \mathrm{H}, \mathrm{Ar}-\mathrm{H}) \\
1.07\left(\mathrm{~s}, 12 \mathrm{H},-\mathrm{CH}_{3}\right) \\
4.13\left(\mathrm{~d}, 8 \mathrm{H},-\mathrm{OCH}_{2}-\right) \\
4.75(\mathrm{~s}, 2 \mathrm{H}, \mathrm{NH}) \\
3.23(\mathrm{t}, 2 \mathrm{H},-\mathrm{CH}) \\
2.10\left(\mathrm{~d}, 4 \mathrm{H},-\mathrm{CH}_{2}\right)\end{array}$ & $\begin{array}{c}22.59\left(\mathrm{CH}_{3}, \mathrm{dtp}\right) \\
36.16(\mathrm{q} \mathrm{C}, \mathrm{dtp}) \\
76.28\left(-\mathrm{OCH}_{2}, \mathrm{dtp}\right) \\
135.34(\mathrm{Ar}-\mathrm{C}) \\
167.56(\mathrm{C}=\mathrm{N}) \\
42.65(\mathrm{CH}) \\
27.30\left(\mathrm{CH}_{2}\right)\end{array}$ & 95.00 \\
\hline 23 & $\mathrm{Zr}_{2}\left(\mathrm{C}_{15} \mathrm{H}_{12} \mathrm{~N}_{2} \mathrm{OCl}\right)_{2}\left[\mathrm{~S}_{6} \mathrm{P}_{2}\left\{\mathrm{OC}\left(\mathrm{CH}_{3}\right)_{2} \mathrm{C}\left(\mathrm{CH}_{3}\right)_{2} \mathrm{O}\right\}_{2}\right]$ & $\begin{array}{c}1.35\left(\mathrm{~s}, 24 \mathrm{H},-\mathrm{CH}_{3}\right) \\
7.76-6.96(\mathrm{~m}, 16 \mathrm{H}, \mathrm{ArH}) \\
4.76(\mathrm{~s}, 2 \mathrm{H},-\mathrm{NH}) \\
3.15(\mathrm{t}, 2 \mathrm{H},-\mathrm{CH}) \\
2.10\left(\mathrm{~d}, 4 \mathrm{H},-\mathrm{CH}_{2}\right)\end{array}$ & $\begin{array}{c}23.59\left(\mathrm{CH}_{3}, \mathrm{dtp}\right) \\
91.85(\mathrm{OC}, \mathrm{dtp}) \\
137.75-123.30(\mathrm{Ar}-\mathrm{C}) \\
167.13(\mathrm{C}=\mathrm{N}) \\
43.23(\mathrm{CH}) \\
27.55\left(\mathrm{CH}_{2}\right) \\
\end{array}$ & 105.00 \\
\hline 24 & 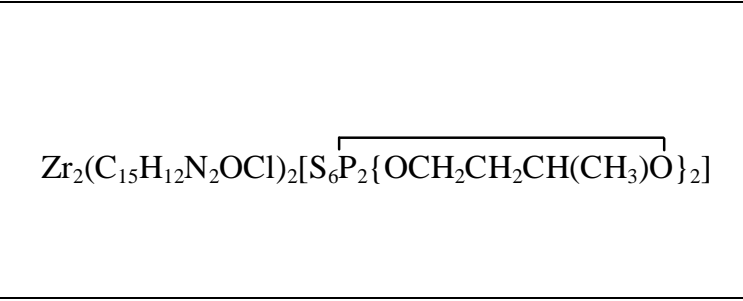 & $\begin{array}{c}2.15-1.23\left(\mathrm{~m}, 10 \mathrm{H},-\mathrm{CH}_{3},\right. \\
\left.\mathrm{CH}_{2}\right) \\
4.10-3.90\left(\mathrm{~m}, 6 \mathrm{H},-\mathrm{OCH}_{2},\right. \\
\mathrm{OCH}) \\
7.45-6.96(\mathrm{~m}, 16 \mathrm{H}, \mathrm{ArH}) \\
5.07(\mathrm{~s}, 2 \mathrm{H},-\mathrm{NH}) \\
3.00(\mathrm{t}, 2 \mathrm{H},-\mathrm{CH}) \\
2.02\left(\mathrm{~d}, 4 \mathrm{H},-\mathrm{CH}_{2}\right)\end{array}$ & $\begin{array}{c}23.45\left(\mathrm{CH}_{3}, \mathrm{dtp}\right) \\
76.29(-\mathrm{OCH}, \mathrm{OCH}, \\
\mathrm{dtp}) \\
136.65-123.45(\mathrm{Ar}-\mathrm{C}) \\
165.15(\mathrm{C}=\mathrm{N}) \\
43.10(\mathrm{CH}) \\
27.29\left(\mathrm{CH}_{2}\right)\end{array}$ & 93.50 \\
\hline
\end{tabular}

Table 4

FAB Mass spectral data for $\mathrm{Zr}_{2}\left(\mathrm{C}_{15} \mathrm{H}_{12} \mathrm{~N}_{2} \mathrm{OX}\right)_{2}\left[\mathrm{~S}_{6} \mathrm{P}_{2}(\mathrm{OR})_{4}\right]$

\begin{tabular}{|c|c|c|c|c|c|c|}
\hline No. & Compound & $\mathrm{m} / \mathrm{z}$ & -py & -2 py & -dtp & -2dtp \\
\hline 3 & $\mathrm{Zr}_{2}\left(\mathrm{C}_{15} \mathrm{H}_{12} \mathrm{~N}_{2} \mathrm{OH}\right)_{2}\left[\mathrm{~S}_{6} \mathrm{P}_{2}\left\{{\left.\left.\mathrm{C}\left(\mathrm{CH}_{3}\right)_{2} \mathrm{CH}_{2} \mathrm{CH}\left(\mathrm{CH}_{3}\right) \mathrm{O}\right)\right]}_{1}\right.\right.$ & 1150 & 913 & 676 & 939 & 728 \\
\hline 9 & $\mathrm{Zr}_{2}\left(\mathrm{C}_{15} \mathrm{H}_{12} \mathrm{~N}_{2} \mathrm{OCH}_{3}\right)_{2}\left[\mathrm{~S}_{6} \mathrm{P}_{2}\left\{\mathrm{OC}\left(\mathrm{CH}_{3}\right)_{2} \mathrm{CH}_{2} \mathrm{CH}\left(\mathrm{CH}_{3}\right) \mathrm{O}\right\}\right]$ & 1175 & 924 & 673 & 964 & 753 \\
\hline 15 & $\mathrm{Zr}_{2}\left(\mathrm{C}_{15} \mathrm{H}_{12} \mathrm{~N}_{2} \mathrm{O}_{2} \mathrm{CH}_{3}\right)_{2}\left(\mathrm{~S}_{6} \mathrm{P}_{2}\left\{\mathrm{OC}\left(\mathrm{CH}_{3}\right)_{2} \mathrm{CH}_{2} \mathrm{CH}\left(\mathrm{CH}_{3}\right) \mathrm{O}\right\}\right]$ & 1255 & 988 & 721 & 1044 & 833 \\
\hline 21 & $\mathrm{Zr}_{2}\left(\mathrm{C}_{15} \mathrm{H}_{12} \mathrm{~N}_{2} \mathrm{OCl}\right)_{2}\left[\mathrm{~S}_{6} \mathrm{P}_{2}\left\{\mathrm{OC}\left(\mathrm{CH}_{3}\right)_{2} \mathrm{CH}_{2} \mathrm{CH}\left(\mathrm{CH}_{3}\right) \mathrm{O}\right\}\right]$ & 1220 & 949 & 678 & 1009 & 798 \\
\hline
\end{tabular}

\subsection{FAB Mass Spectra}

The molecular weights of the synthesized complexes have been determined by FAB mass spectra. The molecular ion peak clearly indicates the dimeric nature of the complexes. FAB mass spectra of all the possible compounds have been carried out but for convenience of presentation and discussion, major fragments of compound number $3,9,15$, and 21 (compounds with different substituted pyrazoline ligands in each series has been selected) have been given (Table 4).

\subsection{XRD and SEM Studies}

These complexes have been examined for crystalline/amorphous nature through XRD. The 
morphology of the complexes was studied by employing XRD, which shows that all the complexes are amorphous solid. Broadening of diffraction peaks was used to estimate the average domain size in terms of "Debeye Scherrer" expression.

$$
\text { Particle size }=D=\frac{0.9 \lambda}{\beta \cos \theta}
$$

where $D$ is the average nanocrystal domain diameter, $\AA ; \beta$ is corrected band broadening (full width at half maxima); $\lambda$ is the $\mathrm{X}$-ray wave length $(1.5418 \AA) ; \theta$ is the diffraction angle.

The value of full width at half maximum intensity $(\beta)$ and corresponding diffraction angle $(\theta)$ is calculated using Fig. 2. The average diameter thus obtained was found to be in the range of $20-30 \mathrm{~nm}$. SEM studies showed that the particle size ranged to about $20 \mathrm{~nm}$. The mean diameter of the different particles synthesized is summarized in Table 5. SEM image and $\mathrm{X}$-ray diffractogram of $\mathrm{Zr}_{2}\left(\mathrm{C}_{15} \mathrm{H}_{12} \mathrm{~N}_{2} \mathrm{OR}\right)_{2}\left[\mathrm{~S}_{6} \mathrm{P}_{2}\left\{\mathrm{OC}\left(\mathrm{CH}_{3}\right)_{2} \mathrm{CH}_{2} \mathrm{CH}\left(\mathrm{CH}_{3}\right) \mathrm{O}\right\}\right.$ are shown in Figs. 1 and 2, respectively.

On the basis of these studies and available literature octahedral geometry may be proposed for $\mathrm{Zr}(\mathrm{IV})$ complexes (Fig. 3).

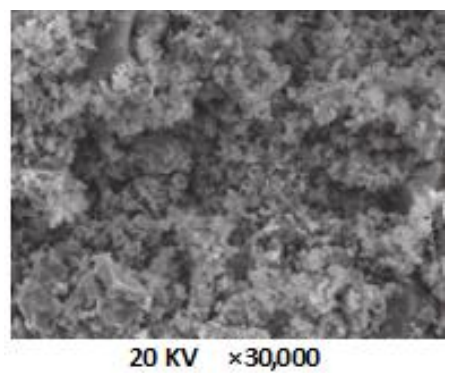

Fig. 1. SEM image

$\mathrm{Zr}_{2}\left(\mathrm{C}_{15} \mathrm{H}_{12} \mathrm{~N}_{2} \mathrm{OH}\right)_{2}\left[\mathrm{~S}_{6} \mathrm{P}_{2}\left\{\mathrm{OC}\left(\mathrm{CH}_{3}\right)_{2} \mathrm{CH}_{2} \mathrm{CH}\left(\mathrm{CH}_{3}\right) \mathrm{O}\right\}\right]$

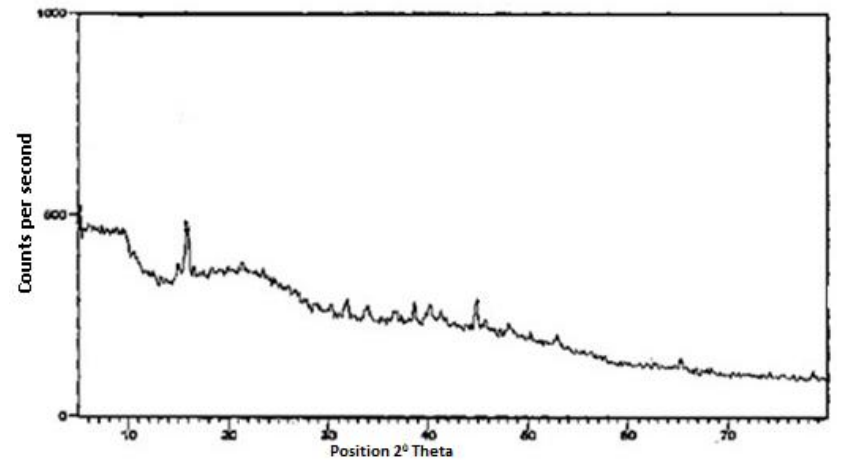

Fig. 2. X-ray diffractogram of $\mathrm{Zr}_{2}\left(\mathrm{C}_{15} \mathrm{H}_{12} \mathrm{~N}_{2} \mathrm{OH}\right)_{2}\left[\mathrm{~S}_{6} \mathrm{P}_{2}\left\{\mathrm{OC}\left(\mathrm{CH}_{3}\right)_{2} \mathrm{CH}_{2} \mathrm{CH}\left(\mathrm{CH}_{3}\right) \mathrm{O}\right\}\right]$

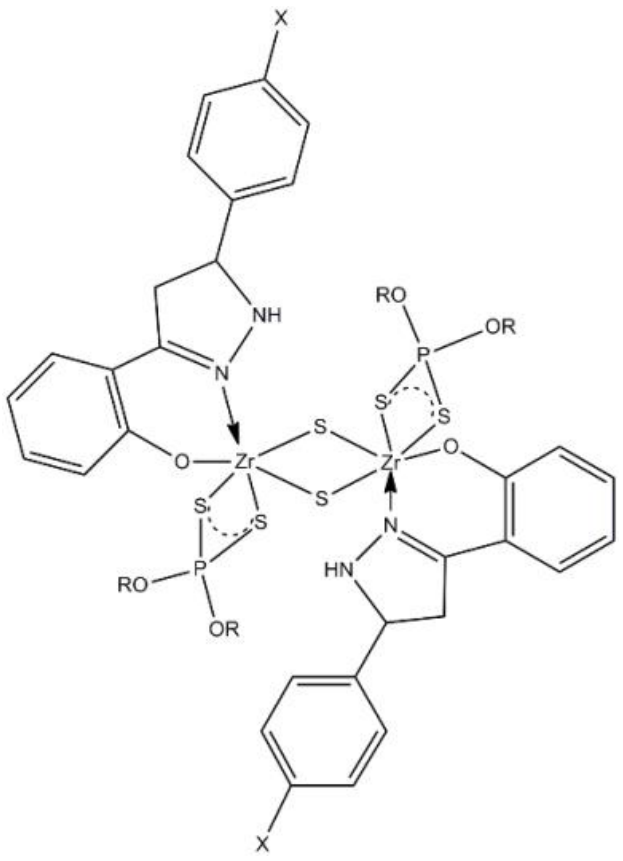

Fig. 3. Proposed structure for $\mathrm{Zr}_{2}\left(\mathrm{C}_{15} \mathrm{H}_{12} \mathrm{~N}_{2} \mathrm{OX}\right)_{2}\left[\mathrm{~S}_{6} \mathrm{P}_{2}(\mathrm{OR})_{4}\right]$

Table 5

Average diameter of particles determined by XRD and SEM

\begin{tabular}{|c|c|c|c|c|}
\hline No. & Compound & $2 \theta$ & $\begin{array}{c}\text { Average diameter } \\
\text { determined by XRD, nm }\end{array}$ & $\begin{array}{c}\text { Average diameter } \\
\text { determined by SEM, } \\
\text { nm }\end{array}$ \\
\hline 3 & $\mathrm{Zr}_{2}\left(\mathrm{C}_{15} \mathrm{H}_{12} \mathrm{~N}_{2} \mathrm{OH}\right)_{2}\left[\mathrm{~S}_{6} \mathrm{P}_{2}\left\{\mathrm{C}\left(\mathrm{CH}_{3}\right)_{2} \mathrm{CH}_{2} \mathrm{CH}\left(\mathrm{CH}_{3}\right) \mathrm{O}\right)\right]$ & 40 & 27 & 17 \\
\hline 9 & $\mathrm{Zr}_{2}\left(\mathrm{C}_{15} \mathrm{H}_{12} \mathrm{~N}_{2} \mathrm{OCH}_{3}\right)_{2}\left[\mathrm{~S}_{6} \mathrm{P}_{2}\left\{\mathrm{OC}\left(\mathrm{CH}_{3}\right)_{2} \mathrm{CH}_{2} \mathrm{CH}\left(\mathrm{CH}_{3}\right) \mathrm{O}\right\}\right]$ & 52 & 33 & 12 \\
\hline 15 & $\mathrm{Zr}_{2}\left(\mathrm{C}_{15} \mathrm{H}_{12} \mathrm{~N}_{2} \mathrm{O}_{2} \mathrm{CH}_{3}\right)_{2}\left(\mathrm{~S}_{6} \mathrm{P}_{2}\left\{\mathrm{OC}\left(\mathrm{CH}_{3}\right)_{2} \mathrm{CH}_{2} \mathrm{CH}\left(\mathrm{CH}_{3}\right) \mathrm{O}\right\}\right]$ & 42 & 23 & 25 \\
\hline 21 & $\mathrm{Zr}_{2}\left(\mathrm{C}_{15} \mathrm{H}_{12} \mathrm{~N}_{2} \mathrm{OCl}\right)_{2}\left[\mathrm{~S}_{6} \mathrm{P}_{2}\left\{\mathrm{OC}\left(\mathrm{CH}_{3}\right)_{2} \mathrm{CH}_{2} \mathrm{CH}\left(\mathrm{CH}_{3}\right) \mathrm{O}\right\}\right]$ & 44 & 19 & 15 \\
\hline
\end{tabular}




\section{Conclusions}

The present study describes the synthesis of a series of complexes of dithiophosphate and pyrazolines with zirconium(IV). The dithiophosphate ligand and pyrazolines behave as bidentate ligand. Elemental analysis data indicate the dimeric nature of the complexes. Octahedral geometry is proposed for the synthesized $\mathrm{Zr}(\mathrm{IV})$ complexes. This class of compound may prove useful precursor for the formation of $\mathrm{ZrS}_{2}$ by sulphide sol gel due to reduced acidity of metal centre. Further studies regarding sulphide sol gel of these derivatives are under investigation.

\section{References}

[1] Peng Y., Krungleviciute V., Eryazici I. et al.: J. Am. Chem. Soc., 2013, 135, 11887. https://doi.org/10.1021/ja4045289

[2] Lee Y., Kim J., Ahm W.: Korean J. Chem. Eng., 2013, 30, 1667. https://doi.org/10.1007/s11814-013-0140-6

[3] Long J., Yaghi O.: Chem. Soc. Rev., 2009, 38, 1213. https://doi.org/10.1039/b903811f

[4] Sharma S., Jain A., Saxena S.: J. Korean Chem. Soc., 2012, 56, 440. https://doi.org/10.5012/jkcs.2012.56.4.440

[5] Bhatt S., Kumari R., Sharma N., Chaudhary S.: Ind. J. Chem. A, 2004, 43, 778 .

[6] Grafov A., Mazurenko E., Battiston G. et al.: J. Phys. IV

France, 1995, 5, C5-497.

https://doi.org/10.1051/jphyscol:1995557

[7] Thomas R., Milanov A., Bhakta R. et al.: Chem.Vap.

Deposition, 2006, 12, 295.

https://doi.org/10.1002/cvde.200506481

[8] Pasko S., Abrutis A., Hubert-Pfalzgraf L.: Mater. Lett., 2005, 59, 261. https://doi.org/10.1016/j.matlet.2004.07.061

[9] Banerjee M., Seidel R., Winter M. et al.: Dalton Trans., 2014, 43, 2384. https://doi.org/10.1039/C3DT52335G

[10] Fleeting K., O'Brien P., Jones A. et al.: J. Chem. Soc., Dalton Trans., 1999, 2853. https://doi.org/10.1039/A901666J

[11] Malghe Y., Prabhu R., Raut R.: Acta. Pol. Pharm., 2009, 66, 45.

[12] Tret'yakova I., Chernii V., Tomachinskaya L. et al.: Theor. Exp. Chem., 2006, 42, 175. https://doi.org/10.1007/s11237-006-0034-3

[13] Bajju G., Devi G., Kotcha S. et al.: Bioinorg. Chem. Appl., 2013, 2013. https://doi.org/10.1155/2013/903616

[14] Kostova I., Momekov G.: Eur. J. Med. Chem., 2006, 41, 717. https://doi.org/10.1016/j.ejmech.2006.03.020

[15] Liu K., Wu Q., Mu X. et al.: Polyhedron, 2013, 52, 222. https://doi.org/10.1016/j.poly.2012.09.044

[16] El- Zoghbi I., Whiyehorne T., Schaper F.: Dalton Trans., 2013, 42, 9376. https://doi.org/10.1039/C2DT31761C

[17] Lenton T., Bercaw J., Panchenko V. et al.: J. Am. Chem. Soc., 2013, 135, 10710. https://doi.org/10.1021/ja403170u

[18] Saha T., Chakraborty D.: Polym. Int., 2013, 62, 1507. https://doi.org/10.1002/pi.4450

[19] Whitelaw E., Jones M., Mahon M., Kociok-Kohn G.: Dalton Trans., 2009, 41, 9020. https://doi.org/10.1039/b911545e [20] Manicone P., Iommetti P., Raffaelli L.: J. Dentistry, 2007, 35, 819. https://doi.org/10.1016/j.jdent.2007.07.008
[21] Denry I., Kelly R.: Dent. Mater., 2008, 24, 299. https://doi.org/10.1016/j.dental.2007.05.007

[22] Yarita T., Aoyagi Y., Sasai H. et al.: Anal. Sci., 2013, 29, 213.

[23] Saxena S., Chakravarty R., Kumar Y. et al.: Separ. Sci.

Technol., 2013, 48, 2108.

https://doi.org/10.1080/01496395.2013.765472

[24] Li W., Deng Q., Fang G. et al.: J. Mater. Chem. B, 2013, 1, 1947. https://doi.org/10.1039/c3tb20127a

[25] Kara A., Tobyn M., Stevens R.: J. Eur. Ceramic Soc., 2004, 24, 3091. https://doi.org/10.1016/j.jeurceramsoc.2003.11.004

[26] Piconi C., Maccauro G.: Biomaterials, 1999, 20, 25. https://doi.org/10.1016/S0142-9612(98)00010-6

[27] ISO TC 150/SC 1 . Implants for surgery ceramic materials based on yttria-stabilized tetragonal zirconia (Y-TZP). ISO/DIS13356, 1995.

[28] von Schewelov T., Sanzen L., Onsten I. et al.: J. Bone Joint Surg. Br., 2005, 87, 1631. https://doi.org/10.1302/0301620X.87B12.16873

[29] Li L., Wang H., Fang X. et al.: Energ. Environ. Sci., 2011, 7, 2586. https://doi.org/10.1039/c1ee01286j

[30] Jang J., Jeong S., Seo J. et al.: J. Am. Chem. Soc., 2011, 133, 7636. https://doi.org/10.1021/ja200400n

[31] Srivastava T.: J. Ind. Chem. Soc., 1981, 58, 710.

[32] Raman N., Raja S., Sakthivel A.: J. Coord. Chem., 2009, 62, 691. https://doi.org/10.1080/00958970802326179

[33] Vogel A.: A Text Book of Qualitative Inorganic Analysis. ELBS Longman Group Ltd, London 1998.

[34] Sharma T., Saxena V., Reddy N.: Acta Chim., 1977, 93, 4. [35] Chauhan H., Chauhan B., Srivastava G., Mehrotra R.:

Phosphorus Sulphur, 1983, 15, 99.

https://doi.org/10.1080/03086648308073283

Received: November 17, 2017 / Revised: December 12, 2017 / Accepted: May 22, 2018

\section{СИНТЕЗ І ХАРАКТЕРИСТИКА ЗМІШАНИХ ЛІГАНДНИХ КОМПЛЕКСІВ ЦИРКОНІЮ(IV) З СУЛЬФУР-, НІТРОГЕН- ТА ОКСИГЕН-ДОНОР ЛІГАНДАМИ}

Анотація. Досліджено новий спосіб синтезу наномірних змішаних лігандних комплексів $\operatorname{Zr}(I V)$ за реакцією хлориду иирконію(IV) з 3(2'-гідроксифеніл)-5-(4-заміщеним феніл) піразолінами та солями дитіофосфату амонію, який після оброблення $\mathrm{H}_{2} \mathrm{~S}$ діє як прекурсор для $\mathrm{ZrS} \mathrm{S}_{2} . \quad$ За допомогою рентгено-дифракційного аналізу досліджено кристалічну/аморфну природу синтезованих комплексів. Визначено, шьо всі комплекси є аморфними твердими речовинами. При визначенні молекулярної маси та проведенні елементарного аналізу і спектроскопічних дослідженнь (IЧ, ${ }^{1}$ Н ЯМР, ${ }^{31}$ Р ЯМР) доведена димерна природа комплексів, в яких дитіофосфат та піразолін є бідентатним лігандом. 3 використанням скануючої електронної мікроскопї показано, щзо частинки знаходяться в нанодіапазоні. Запропоновано координаційне число 6 для цирконію з восьмигранною геометрією.

Ключові слова: циирконій(IV), дитіофосфат, піразолін, наночастинка, соль-гель. 